\title{
Numbers and functions in quantum field theory
}

\author{
Oliver Schnetz \\ Department Mathematik, Friedrich Alexander Universität Erlangen-Nürnberg, \\ Cauerstraße 11, 91058 Erlangen, Germany
}

(Received 31 January 2018; published 23 April 2018)

\begin{abstract}
We review recent results in the theory of numbers and single-valued functions on the complex plane which arise in quantum field theory. These results are the basis for a new approach to high-loop-order calculations. As concrete examples, we provide scheme-independent counterterms of primitive logdivergent graphs in $\phi^{4}$ theory up to eight loops and the renormalization functions $\beta, \gamma, \gamma_{m}$ of dimensionally regularized $\phi^{4}$ theory in the minimal subtraction scheme up to seven loops.
\end{abstract}

DOI: 10.1103/PhysRevD.97.085018

\section{INTRODUCTION}

Quantum field theories (QFTs) are fundamental theories of physical interactions. Physical QFTs are the electroweak theory which combines electromagnetism with the weak interaction, quantum chromodynamics which describes the interaction between quarks and gluons, and $\phi^{4}$ theory for the Higgs boson. Gravity has not yet found a quantum formulation.

Although QFTs are experimentally very well confirmed (see e.g. the anomalous magnetic moment of the electron for an impressive example, Sec. IV J [1-4]), a complete mathematical understanding of QFTs is lacking. On the one hand, there are fundamental questions like the existence and structure of QFTs. On the other hand, there is demand for practical tools to perform QFT calculations. Due to the mathematical difficulty of QFTs progress is modest. Here, we report on some recent results in both directions.

For a while it seemed possible that the number content of QFT is given by multiple zeta values (MZVs) which are multiple sums that generalize the Riemann zeta function at positive integer arguments. Assuming standard conjectures it has now been proved that this is not the case $[5,6]$.

With the theory of graphical functions, a tool was developed to perform multiloop calculations in massless scalar field theories [7-9]. A first notable breakthrough was the proof of the zig-zag conjecture $[10,11]$ which

\footnotetext{
*schnetz@mi.uni-erlangen.de
}

Published by the American Physical Society under the terms of the Creative Commons Attribution 4.0 International license. Further distribution of this work must maintain attribution to the author(s) and the published article's title, journal citation, and DOI. Funded by SCOAP ${ }^{3}$. gives an explicit formula for periods ${ }^{1}$ of zig-zag graphs (see Theorem 4.8).

For more general applications it was necessary to introduce a novel family of single-valued functions on the complex plane: generalized single-valued hyperlogarithms (GSVHs, see Sec. IV D 7). Generalized single-valued hyperlogarithms vastly generalize single-valued multiple polylogarithms. Nevertheless it was possible to translate the vital properties of single-valued multiple polylogarithms into the framework of GSVHs [13]. A Maple ${ }^{\mathrm{TM}}$ package was developed that can calculate many periods in $\phi^{4}$ theory up to 11 loops [14]. With a large amount of data available, the structure of $\phi^{4}$ periods could be connected to the Galois theory of algebraic integrals [15-17].

To make further contact to physics, it is necessary to regularize integrals which diverge in four dimensions. This is often done by generalizing to $4-\epsilon$ "dimensions" (which can be defined in a parametric representation of QFT integrals [18]). Using GSVHs it was possible to obtain $\epsilon$-expansions for QFT periods and graphical functions. The procedure Phi4 in HyperlogProcedures calculates the $\beta$-function and the anomalous dimensions $\gamma$ and $\gamma_{m}$ up to seven loops in the minimally subtracted $O(n)$ symmetric $\phi^{4}$ theory [14]. The self-energy can be calculated to six loops (see Sec. IV D 9 for results in the case $n=1$ ).

\footnotetext{
${ }^{1}$ We use the word "period" for numbers that arise in perturbative quantum field theory. The name comes from [12], where periods are defined as numbers which are obtained from algebraic integrals (defined using polynomials with integer coefficients). Note that the name period in mathematics has only a small overlap with the physical notion of a period. In the concrete case of primitive log-divergent Feynman graphs (as in the zig-zag conjecture or in Table III), periods are the (scheme-independent) residues of the regularized Feynman integrals. Also the coefficients of the $\phi^{4}$ renormalization functions in Sec. IV D 9 are periods.
} 


\section{GENERAL IDEA}

In QFT, graphs are used to symbolize integrals. One first has to fix a space-time dimension $d$ which, for brevity, we connect to the parameter $\lambda$ according to

$$
d=2+2 \lambda>2 .
$$

The chosen QFT determines which graphs are admissible. Here we are mainly interested in $\phi^{4}$ theory which limits the vertex degree to four. Feynman rules translate graphs to integrals. They exist in momentum and in position space with integration over $d$-dimensional variables associated to independent cycles or vertices, respectively. For massive theories one needs to use momentum space to obtain explicitly algebraic integrands. Here we are mostly interested in massless calculations which allows us to use position space.

The general setup is as follows. Assume $G$ is a graph with edges $\mathcal{E}(G)$ and vertices $\mathcal{V}(G)$. We do not assume here that every vertex in $G$ has maximum degree four. Every edge $e \in \mathcal{E}(G)$ has a weight $\nu_{e} \in \mathbb{R}$. We assume that $G$ has no self-loops (tadpoles). This is common in dimensionally regularized massless theories. The edge weight is additive, i.e. a multiple edge in $G$ is equivalent to a single edge with weight equal to the weight sum of the multiple edge. Therefore, we only need to consider single edges. We split the set of vertices into 'internal' vertices $\mathcal{V}^{\text {int }}(G)$ and 'external' vertices $\mathcal{V}^{\operatorname{ext}}(G)$. To every vertex we associate a $d$ dimensional variable and do not distinguish between the vertex and the variable. We use $x_{i}, i=1, \ldots, V^{\text {int }}(G)=$ $\left|\mathcal{V}^{\text {int }}(G)\right|$ for internal vertices and $z_{i}, i=1, \ldots, V^{\operatorname{ext}}(G)=$ $\left|\mathcal{V}^{\text {ext }}(G)\right|$ for external vertices. To every edge $e=\{u, v\} \in$ $\mathcal{E}(G)$ between the two vertices $u, v \in \mathcal{V}(G)$ (internal or external) we associate a quadric $Q_{e}$ which is given by the Euclidean distance between (the variables associated to) $u$ and $v$,

$$
Q_{e}(u, v)=\|u-v\|^{2}=\left(u_{1}-v_{1}\right)^{2}+\cdots+\left(u_{d}-v_{d}\right)^{2} .
$$

Assume the graph $G$ has the property that the following integral exists

$f_{G}^{(\lambda)}\left(z_{1}, \ldots, z_{V^{\text {ext }}}\right)=\left(\prod_{v=1}^{V^{\text {int }}(G)} \int_{\mathbb{R}^{d}} \frac{\mathrm{d}^{d} x_{v}}{\pi^{d / 2}}\right) \frac{1}{\prod_{e \in \mathcal{E}(G)} Q_{e}^{\lambda \nu_{e}}}$.

Due to translational and scale invariance the integral can only exist if $G$ has at least two external vertices. In the case of exactly two external vertices, the integral is determined up to a constant by these symmetries,

$$
f_{G}^{(\lambda)}\left(z_{1}, z_{2}\right)=P(G)\left\|z_{1}-z_{2}\right\|^{d V^{\operatorname{int}(G)}-2 \lambda \sum_{e \in \mathcal{E}(G)} \nu_{e} .}
$$

The number $P(G) \in \mathbb{R}_{+}$is the Feynman period of $G$.
In primitive logarithmically divergent physical graphs (with external legs), the residue (in the regulator) is given by the period of the graph with amputated external legs. Therefore the calculation of periods is of great importance for renormalizing QFTs (see e.g. [10]).

Without loss of information, we set $z_{1}={ }^{\prime} 0{ }^{\prime}=(0, \ldots, 0)$ and $z_{2}={ }^{\prime} 1$ ' $=(1,0, \ldots, 0)$ (we may associate to the vertex 1 any unit vector in $\left.\mathbb{R}^{d}\right)$ and obtain

$$
f_{G}^{(\lambda)}(0,1)=P(G)
$$

In the case of three external vertices, we can again exploit the symmetry of the integral to reduce the number of variables. In this case, we may use a complex variable $z$ (and its complex conjugate $\bar{z}$ ) to describe the functional behavior of $f_{G}^{(\lambda)}$. We obtain the "graphical function" (which we also give the symbol $f_{G}^{(\lambda)}$ ) [9]

$$
f_{G}^{(\lambda)}(z)=f_{G}^{(\lambda)}\left(0,1,\left(\frac{z+\bar{z}}{2}, \frac{z-\bar{z}}{2 \mathrm{i}}, 0, \ldots, 0\right)\right) .
$$

In full generality, $f_{G}^{(\lambda)}(z)$ is a positive single-valued real analytic function on $\mathbb{C} \backslash\{0,1\}$ [8] with the residual symmetry

$$
f_{G}^{(\lambda)}(z)=f_{G}^{(\lambda)}(\bar{z})
$$

The benefit of complex numbers is that quadrics between external vertices factorize

$Q_{\{0,1\}}=1, \quad Q_{\{0, z\}}=z \bar{z}, \quad Q_{\{1, z\}}=(z-1)(\bar{z}-1)$.

General graphs with four or more external vertices lead to functions which effectively depend on a variable in $\mathbb{R}^{3}$. Such functions do not have this factorizing property. An exception are "conformal" graphs with four external vertices where every internal vertex has degree $2 d /(d-2)$. In this case, one may use an inversion $x_{i} \mapsto x_{i} /\left\|x_{i}\right\|^{2}$ to reduce the integral to the case of three external vertices e.g. $[7,19]$. Here, we go the opposite direction and 'complete' graphical functions to conformal graphs with four external vertices [9]. We will see in Sec. IV D 1 that this is useful to exploit the full symmetry of graphical functions.

In the following, we restrict ourselves to the above two cases, periods and graphical functions. In $\phi^{4}$ theory, fourpoint functions are formally conformal. However, only the tree level contribution is convergent. A practical tool to resolve divergences is to transform all integrals in a parametric form using the Schwinger trick [18]. In parametric form, one integrates over one-dimensional variables associated to the edges of the graph. The dimension $d$ enters the integrand as an exponent. It is hence possible to consider $d$ as a parameter and use analytic continuation to $d=4-\epsilon$ (losing conformal invariance). All parametric 
integrals have Laurent expansions at $\epsilon=0$. Graphical functions are amendable to such a procedure. A general parametric representation of graphical functions is given in [8] (which generalizes a formula in [20], see also [21] as a general reference for the parametric representation of Feynman integrals).

\section{NUMBERS}

A graph $G$ is called $\phi^{4}$ if $G$ has maximum vertex degree four. The period of a $\phi^{4}$ graph in $d=4$ dimensions is a $\phi^{4}$ period. The loop order of $G$ is the number of independent cycles in $G$.

\section{A. Completion}

We "complete" a graph $G$ with two external vertices 0 and 1 by adding a new external vertex which we give the label ' $\infty$ ' (as reference to conformal symmetry) [22]. We add edges from $\infty$ to all internal vertices in $G$ such that every internal vertex has degree $2 d /(d-2)$. Finally, we add a weighted triangle with vertices $0,1, \infty$ such that the completed graph becomes $2 d /(d-2)$ regular. We denote the completion of $G$ by $\bar{G}$. It is easy to see that completion is always possible and unique. If we employ the Feynman rule that every edge $e$ adjacent to $\infty$ has quadric $Q_{e}=1$ we find that the period (1) does not change under completion.

The power of completion is that the period of a completed graph does not depend on the choice of the external vertices $0,1, \infty$. This was proved in four dimensions in Theorem and Definition 2.7 in [22]. The $d$-dimensional case is strictly analogous. Hence, in $\bar{G}$ we do not need the distinction between internal and external vertices. We, henceforth, consider $\bar{G}$ as an unlabeled graph.

Obviously, different graphs $G_{1}$ and $G_{2}$ can have the same completion. In this case, completion implies equality of their periods: $P\left(G_{1}\right)=P\left(G_{2}\right)$ if $\overline{G_{1}}=\overline{G_{2}}$. This identity on periods was already used in [10]. Completion is effectively a tool to organize equivalence classes of graphs with identical period. We define $P(\bar{G}):=P(G)$ if $\bar{G}$ is the completion of $G$. The loop order of a completed graph $\bar{G}$ is defined as the number of independent cycles in the uncompleted graph $G$. So, by definition, completion does not change the loop order.

\section{B. Existence}

In four dimensions, the period of a completed graph $\bar{G}$ with edge-weights 1 exists if and only if $\bar{G}$ is internally 6 -connected. This means that the only way to cut $\bar{G}$ with less than 6 edge cuts is to separate off a vertex (Proposition 2.6 in [22]).

In general, a Feynman period $P(G)$ can be considered as a graphical function (see Sec. IV D 1) with an isolated external vertex $z$. Existence of $P(G)$ in general is hence a special case of the criterion for the existence of graphical functions in Theorem 4.3.

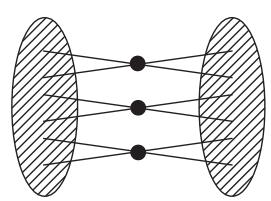

$\mathrm{P}(\overline{\mathrm{G}})$

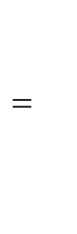

$=$

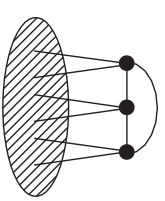

$x$

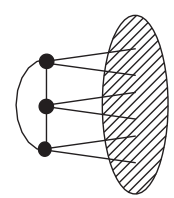

$\mathrm{P}\left(\overline{\mathrm{G}}_{1}\right) \quad \mathrm{P} \quad \mathrm{P}\left(\overline{\mathrm{G}}_{2}\right)$
FIG. 1. Vertex connectivity 3 leads to a product of periods.

A completed graph with existing period in four dimensions is called completed primitive [22].

\section{Product identity}

The period of a completed graph $\bar{G}$ can only exist if $\bar{G}$ has vertex connectivity $\geq 3$ (the vertex connectivity is the minimum number of vertices which, when removed, split the graph). The period of a completed graph $\bar{G}$ with vertexconnectivity three factorizes in the way depicted in Fig. 1. Reversely, completed primitive graphs $\bar{G}_{1}, \bar{G}_{2}$ with triangles can be glued along triangles to provide a completed graph with period $P\left(\bar{G}_{1}\right) P\left(\bar{G}_{2}\right)$. The case $d=4$ with unit edge-weights was treated in Theorem 2.10 in [22]. The general case (where the weights of the triangles follow from $2 d /(d-2)$ regularity) is analogous.

A completed graph with vertex connectivity three is called reducible, otherwise it is irreducible [22]. A list of all irreducible completed primitive graphs up to eight loops (in four dimensions with unit edge weights) is given in Table III at the end of this report. Hyperlog Procedures extends this list to eleven loops [14].

Because not all completed primitive graphs have triangles (see e.g. $P_{6,4}$ in Table III) it is unclear if, in general, the product of Feynman periods is a Feynman period. In particular, one may ask if the $\mathbb{Z}$-span of $\phi^{4}$ periods is a ring (or-weaker-if $\phi^{4}$ periods span a $\mathbb{Q}$ algebra).

\section{Twist and Fourier identity}

There exist two more known identities on $\phi^{4}$ periods. The (for graphs with many vertices) frequent twist identity and the rare Fourier identity [22]. The Fourier identity was already used in [10]. The first example of a twist identity which is not also explained by a Fourier identity appears at eight loops. Twist and Fourier identities are listed in Table III.

Recent results on the Hepp invariant seem to indicate that more identities between periods exist (see Sec. III C 8).

\section{E. The number content of $\phi^{\mathbf{4}}$ periods}

Up to five loops there exists at most one $\phi^{4}$ period per loop order. These periods are the first instances of the infinite family of zig-zag periods (see Fig. 5). The periods of the zig-zag family are rational multiples of the Riemann zeta function at odd arguments (see Theorem 4.8). 


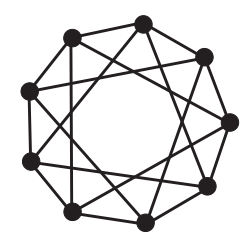

FIG. 2. The completed graph $P_{7,11}$.

At six loops the $\phi^{4}$ periods $P_{6,3}$ and $P_{6,4}$ have a zeta double sum of weight eight. In general, Feynman periods are often multiple zeta values (MZVs) which are $\mathbb{Q}$ linear combinations of multiple zeta sums

$\zeta\left(n_{d}, \ldots, n_{1}\right)=\sum_{k_{d}>\ldots>k_{1} \geq 1} \frac{1}{k_{d}^{n_{d}} \cdots k_{1}^{n_{1}}}$ with $n_{i} \in \mathbb{Z}_{>0}, \quad n_{d} \geq 2$.

At seven loops there exists a single period, $P_{7,11}$, which (conjecturally) is not expressible in terms of MZVs. The period $P_{7,11}$ (see Fig. 2) features an extension of MZVs by (some) sixth (or third) roots of unity. We give the result in the $f^{6}$ alphabet with respect to the corresponding even parity Deligne basis [17] (a presentation of $P_{7,11}$ with smaller numerators and denominators is given in [31]):

$$
\begin{aligned}
\frac{P_{7,11}}{\mathrm{i} \sqrt{3}}= & -\frac{332262}{43} f_{8}^{6} f_{3}^{6}+\frac{54918}{55} f_{6}^{6} f_{5}^{6}+\frac{1134}{13} f_{4}^{6} f_{7}^{6} \\
& -\frac{1874502}{3485} f_{2}^{6} f_{9}^{6}-5670 f_{2}^{6} f_{3}^{6} f_{3}^{6} f_{3}^{6} \\
& -\frac{3216912825399005402331281812377062149}{10264478246467100965990650592350882000} \\
& \times(\pi \mathrm{i})^{11} .
\end{aligned}
$$

There exists a lengthy conversion of the period $P_{7,11}$ in terms of multiple polylogarithms evaluated at primitive sixth roots of unity. The period $P_{7,11}$ was calculated by Erik Panzer (using his program HyperInt) in his $\mathrm{PhD}$ thesis [23] in terms multiple polylogarithms. With HyperlogProcedures the result was converted into the $f$ alphabet [14].

At eight loops still most periods are MZVs. Beyond MZVs we found for the period $P_{8,33}$ an expression of weight 13 which is similar to $P_{7,11}$. Moreover, there exist four periods of an entirely new type. The geometry underlying these periods is no longer a punctured sphere $\mathbb{C} \backslash\{0,1, \ldots\}$. Instead of point punctures, we obtain in two cases K3 surfaces [6]. In the other two cases, we found a threefold and a fivefold, respectively. All four varieties are modular of low level [32].

Beyond eight loops periods associated to nonmodular varieties are expected [32].

\section{F. The coaction conjectures}

In (3), a letter $f^{6}$ of even weight (subscript) appears only in the leftmost position. This is a consequence of a Galois structure in $\phi^{4}$ periods.
In [12], M. Kontsevich and D. Zagier defined the $\mathbb{Q}$ algebra of periods $\mathcal{P}$ as integrals of rational forms over $\mathbb{Q}$. Feynman periods are periods in this sense. By general philosophy there should exist a Galois coaction on $\mathcal{P}$ [16,33-38],

$$
\Delta: \mathcal{P} \rightarrow \mathcal{P}^{\mathfrak{d r}} \otimes_{\mathbb{Q}} \mathcal{P},
$$

where the left-hand side of the tensor product is the Hopf algebra of de Rham periods. (Note that in some publications $\mathcal{P}^{\text {dr }}$ coacts on the right-hand side.) In the special case of $\phi^{4}$ periods, the right-hand side (and also the left-hand side) of (4) seems to be severely restricted. A mathematical theory with first results is in [15]. The data of approximately 300 known $\phi^{4}$ periods up to 11 loops led to the following possible scenarios [17] for the $\mathbb{Q}$ algebra $\mathcal{P}_{\phi^{4}}$ generated by $\phi^{4}$ periods. (More precisely, in this article $\Delta$ is the unipotent part of the coaction.)

Scenario 1.

$$
\Delta: \mathcal{P}_{\phi^{4}} \rightarrow \mathcal{P}^{\mathfrak{d r}} \otimes_{\mathbb{Q}} \mathcal{P}_{\phi^{4}}
$$

Scenario 2.

$$
\Delta^{\prime}: \mathcal{P}_{\phi^{4}, \leq n} \rightarrow \mathcal{P}^{\mathfrak{d r}} \otimes_{\mathbb{Q}} \mathcal{P}_{F, \leq n-1},
$$

where

$$
\Delta^{\prime} x=\Delta x-1 \otimes x
$$

is the reduced coaction.

Scenario 2 means that for a given $\phi^{4}$ period of $n$ loops the right-hand side of the tensor product is in the $\mathbb{Q}$ vector space spanned by Feynman periods of all graphs with at most $n-1$ loops.

Note that $\mathcal{P}_{\phi^{4}}$ and $\mathcal{P}_{F}$ are very sparse in $\mathcal{P}$, so that the Scenarios 1 and 2 have huge predictive power on $\mathcal{P}_{\phi^{4}}$. Presumably $\mathcal{P}_{\phi^{4}}$ also is very sparse in $\mathcal{P}_{F}$. The coaction conjectures are (5) and (6).

\section{G. The $c_{2}$ invariant}

The $c_{2}$ invariant assigns to every graph with at least three vertices an infinite sequence which is indexed by prime powers $q=p^{n}$ [39],

$$
\begin{aligned}
c_{2}: G & \mapsto\left(c_{2}(G)_{q}\right)_{q} \\
& =\left(c_{2}(G)_{2}, c_{2}(G)_{3}, c_{2}(G)_{4}, c_{2}(G)_{5}, c_{2}(G)_{7}, \ldots\right),
\end{aligned}
$$

where $c_{2}(G)_{q}$ is in $\mathbb{Z} / q \mathbb{Z}$. The $c_{2}$ invariant is linked to the period integral in four dimensions. With a metric signature $(+,-,+,-)$ instead of the Euclidean signature it can be defined via the point-count $N_{q}$ of the singular locus of the period integral (1) over the finite field $\mathbb{F}_{q}[40,41]$. For graphs with at least three vertices, $N_{q}$ is divisible by $q^{2}$ and we define 
TABLE I. List of $\phi^{4}$-transcendentals up to loop-order eight. The list is incomplete at loop-order eight.

\begin{tabular}{|c|c|c|c|}
\hline$\ell$ & Weight & Number & Value \\
\hline 1 & 0 & $Q_{0}=1$ & 1 \\
\hline 3 & 3 & $Q_{3}=\zeta(3)$ & 1.202056903159 \\
\hline 4 & 5 & $Q_{5}=\zeta(5)$ & 1.036927755143 \\
\hline 5 & 7 & $Q_{7}=\zeta(7)$ & 1.008349277381 \\
\hline \multirow[t]{2}{*}{6} & 8 & $Q_{8}=N_{3,5}$ & 0.070183206556 \\
\hline & 9 & $Q_{9}=\zeta(9)$ & 1.002008392826 \\
\hline \multirow[t]{4}{*}{7} & 10 & $Q_{10}=N_{3,7}$ & 0.090897338299 \\
\hline & 11 & $Q_{11,1}=\zeta(11)$ & 1.000494188604 \\
\hline & & $Q_{11,2}=-\zeta(3,5,3)+\zeta(3) \zeta(5,3)$ & 0.042696696025 \\
\hline & & $Q_{11,3}=P_{7,11}$, Eq. (3) & 200.357566429 \\
\hline \multirow[t]{7}{*}{8} & 12 & $Q_{12,1}=N_{3,9}$ & 0.096506102637 \\
\hline & & $Q_{12,2}=N_{5,7}$ & 0.020460547937 \\
\hline & & $Q_{12,3}=\pi^{12} / 10 !$ & 0.254703808841 \\
\hline & 13 & $Q_{13,1}=\zeta(13)$ & 1.000122713347 \\
\hline & & $Q_{13,2}=-\zeta(5,3,5)+11 \zeta(5) \zeta(5,3)+5 \zeta(5) \zeta(8)$ & 5.635097688692 \\
\hline & & $Q_{13,3}=-\zeta(3,7,3)+\zeta(3) \zeta(7,3)+12 \zeta(5) \zeta(5,3)+6 \zeta(5) \zeta(8)$ & 6.725631947085 \\
\hline & & $Q_{13,4}=P_{8,33}[14]$ & 468.038498992 \\
\hline
\end{tabular}

TABLE II. Conversion of the $N_{a, b} \mathrm{~s}$ in Table I into MZVs.

\begin{tabular}{|c|c|c|}
\hline$\ell$ & Weight & Base \\
\hline 6 & 8 & $N_{3,5}=\frac{27}{80} \zeta(5,3)+\frac{45}{64} \zeta(5) \zeta(3)-\frac{261}{320} \zeta(8)$ \\
\hline 7 & 10 & $N_{3,7}=\frac{423}{3584} \zeta(7,3)+\frac{189}{256} \zeta(7) \zeta(3)+\frac{639}{3584} \zeta(5)^{2}-\frac{7137}{7168} \zeta(10)$ \\
\hline 8 & 12 & $\begin{aligned} N_{3,9}= & \frac{27}{512} \zeta(4,4,2,2)+\frac{55}{1024} \zeta(9,3)+\frac{231}{256} \zeta(9) \zeta(3)+\frac{447}{256} \zeta(7) \zeta(5)-\frac{9}{512} \zeta(3)^{4}-\frac{27}{448} \zeta(7,3) \zeta(2)-\frac{189}{128} \zeta(7) \zeta(3) \zeta(2) \\
& -\frac{1269}{1792} \zeta(5)^{2} \zeta(2)+\frac{189}{512} \zeta(5,3) \zeta(4)+\frac{945}{512} \zeta(5) \zeta(3) \zeta(4)+\frac{9}{64} \zeta(3)^{2} \zeta(6)-\frac{7322453}{5660672} \zeta(12) \\
N_{5,7}= & -\frac{81}{512} \zeta(4,4,2,2)+\frac{19}{1024} \zeta(9,3)-\frac{477}{1024} \zeta(9) \zeta(3)-\frac{4449}{1024} \zeta(7) \zeta(5)+\frac{27}{512} \zeta(3)^{4}+\frac{81}{448} \zeta(7,3) \zeta(2)+\frac{567}{128} \zeta(7) \zeta(3) \zeta(2) \\
& +\frac{3807}{1792} \zeta(5)^{2} \zeta(2)-\frac{567}{512} \zeta(5,3) \zeta(4)-\frac{2835}{512} \zeta(5) \zeta(3) \zeta(4)-\frac{27}{64} \zeta(3)^{2} \zeta(6)+\frac{3155095}{5660672} \zeta(12)\end{aligned}$ \\
\hline
\end{tabular}

$$
c_{2}(G)_{q} \equiv N_{q} / q^{2} \bmod q
$$

In practice, it is more efficient to calculate the $c_{2}$ invariant in parametric space where the above equation is still valid $[6,39]$.

The power of the $c_{2}$ invariant is twofold: First, there exist powerful tools which make it possible to determine the $c_{2}$ invariant for many graphs. If the $c_{2}$ invariant cannot be fully calculated, it is still possible to determine the $c_{2}$ invariant for small primes $[32,42]$. For all completed primitive $\phi^{4}$ graphs up to ten loops the $c_{2}$ is known for at least the first six primes (in most cases much more) [32].

Second, the $c_{2}$ invariant has some predictive power for the period. In particular, if two graphs have the same period, they are conjectured to have the same $c_{2}$ invariant,

$$
P\left(G_{1}\right)=P\left(G_{2}\right) \Rightarrow c_{2}\left(G_{1}\right)=c_{2}\left(G_{2}\right) .
$$

All graphs with $c_{2}$ invariant -1 (i.e. $c_{2}(G)_{q} \equiv-1 \bmod q$ for all $q$ ) should have an MZV period. If the $c_{2}$ invariant is $-z_{2}$, with

$$
z_{N}(q)= \begin{cases}1 & \text { if } N \mid q-1 \\ 0 & \text { if } \operatorname{gcd}(N, q)>1 \\ -1 & \text { otherwise }\end{cases}
$$

then the period is expected to be an Euler sum (due to the coaction conjectures, in many cases these periods are still MZVs [17]). The $c_{2}$ invariant of the periods $P_{7,11}$ and $P_{8,33}$ are $-z_{3}$. This links the $c_{2}$ invariant to the sixth roots of unity which exist in (3).

The connection between $\phi^{4}$ periods and higher dimensional geometries (in some graphs with at least eight loops) is proved with the $c_{2}$ invariant [6,32].

This led to the proof (assuming standard transcendentality conjectures) that not all $\phi^{4}$ periods are MZVs or extensions of MZVs by algebraic numbers $[5,6]$. Concretely, it was shown that $P_{8,37}$ is linked in such a way to the geometry of a $\mathrm{K} 3$ surface (which is modular of weight 3 level 7) that the "motivic" period cannot be mixed Tate.

The $c_{2}$ invariant can also be zero (i.e. $c_{2}(G)_{q} \equiv 0 \bmod q$ for all $q$ ). In this case, the period (conjecturally) has 'weight 
drop'. This means that the transcendental weight of the period is strictly smaller than the maximum value $2 \ell-3$ in loop order $\ell$.

Note that the $c_{2}$ invariant seems very sparse for $\phi^{4}$ periods. It can be conjectured that for any fixed dimension (of the lowest dimensional manifold whose point-count gives the $c_{2}$ ) there exist only finitely many $c_{2} \mathrm{~s}$ to all loop orders. For dimension 0 we have $-z_{N}$ for $N=1,2,3$, 4. In dimension one, there seems to be nothing. We possibly only have three two-folds (which are modular K3s of low level) and five modular three-folds in $\phi^{4}$ [32].

This is in stark contrast to the situation in $\mathcal{P}_{F}$ (including non- $\phi^{4}$ graphs) where we expect that we see basically any geometry over $\mathbb{Z}$ in the $c_{2}$.

An interesting recent result by $\mathrm{K}$. Yeats (private communication 2017) is that we seem to see any finite sequence of prime remainders in the $c_{2}$ of $\phi^{4}$ graphs. Note that this does not contradict the sparsity of $\phi^{4} c_{2}$ s (because $c_{2}$ s are infinite sequences).

\section{H. The Hepp invariant}

For any graph $G$ with edge weights $\left\{\nu_{e}\right\}, e \in \mathcal{E}(G)$ and $a \in \mathbb{C}$ we recursively define the following Hepp invariant:

$$
H_{a}(G)=\frac{\sum_{e \in \mathcal{E}(G)} \nu_{e} H_{a}(G \backslash e)}{N_{G}-a h_{1}(G)}
$$

where $N(G)=\sum_{e \in \mathcal{E}(G)} \nu_{e}$ is the sum of edge weights and $h_{1}(G)$ is the (Betti) number of independent cycles in $G$. A graph with no edges has Hepp invariant 1.

Lemma 3.1. The Hepp invariant has the following properties:

(1) If the removal of the edge $e$ disconnects the graph $G$ then $H_{a}(G)=H_{a}(G \backslash e)$.

(2) If $\mathrm{G}$ has vertex connectivity $\leq 1$ then $H_{a}$ factorizes, i.e. $H_{a}(G)$ is the product of the Hepp invariants of its components (cutting at split vertices without removing edges).

(3) Assume a vertex $v$ in $G$ is adjacent to exactly two edges $e$ and $f$ with weights $\nu_{e}$ and $\nu_{f}$. We construct a smaller graph $G^{\prime}$ by contracting the edge $e$ (or $f$ ) in $G$ and giving $f(e)$ the weight $\nu_{e}+\nu_{f}$. Then $H_{a}(G)=H_{a}\left(G^{\prime}\right)$.

Proof.-Straightforward induction over the number of edges in $G$.

The above lemma can be used to efficiently calculate the Hepp invariant of reasonably large graphs. Note that every forest has Hepp invariant 1.

If a graph $G$ has a Feynman period in $d=4$ dimensions then $H_{a}(G)$ trivially has a simple pole at $a=2$. We define the Hepp period of $G$ as the residue,

$$
H(G)=-h_{1}(G) 2^{-h_{1}(G)} \operatorname{res}_{a=2} H_{a}(G) .
$$

Erik Panzer recently found that the Hepp period is closely related to the Feynman period $[30,43]$. It approximates the Feynman period surprisingly well,

$$
P(G) \approx 0.545^{h_{1}(G)-1} H(G)^{1.355} .
$$

with an error of a few percent. The numerical estimates for the unknown eight loop periods in Table III were obtained by a refinement of (10).

Even more surprisingly, the Hepp period seems to know all identities between periods.

Conjecture 3.2. (E. Panzer).

$$
P\left(G_{1}\right)=P\left(G_{2}\right) \Leftrightarrow H\left(G_{1}\right)=H\left(G_{2}\right)
$$

At eight loops, the above conjecture requires $P_{8,30}=$ $P_{8,36}$ and $P_{8,31}=P_{8,35}$. These identities do not follow from twists or Fourier identities.

Assuming Conj. 3.2 for completion, the product identity (Sec. III C 3) was proved by E. Panzer (private communication) and independently by K. Yeats (private communication) to hold for Hepp periods.

It is easy to see that $P(G) \leq 2^{h_{1}(G)} H(G)$ which is the classical Hepp bound for periods. Erik Panzer has achieved a certain refinement of this bound [30]. We conjecture that the Hepp period is a (crude) upper bound for the period, $P(G) \leq H(G)$.

\section{FUNCTIONS}

Let $G$ be a graph with external vertices $0,1, z$ such that the graphical function $f_{G}^{(\lambda)}$ exists.

\section{A. Completion}

Like in the case of periods, completion exploits conformal symmetry to handle equivalence classes of closely related graphical functions. Again, we add an external vertex ' $\infty$ ' which connects to all internal vertices of $G$ with weights that give the internal vertices weighted degree $2 d /(d-2)$. Now, we add edges $\{z, \infty\},\{0,1\},\{0, \infty\}$, $\{1, \infty\}$ such that all external vertices have weighted degree 0 . This provides the completion $\bar{G}$ of $G$. The graphical function $f_{\bar{G}}^{(\lambda)}$ of the completed graph $\bar{G}$ is defined as the graphical function of $\bar{G} \backslash \infty$ (edges adjacent to $\infty$ have quadric 1). Clearly, the graphical function does not change under completion. Completion is always possible and unique. In three or four dimensions, we also know that the completed graph $\bar{G}$ has integer edge weights if $G$ has (Lemma 3.18 in [9]).

A permutation of external vertices in a completed graph results in a Möbuis transformation of the argument $z$ :

Theorem 4.1. (Theorem 3.20 in [9]). Let $\sigma:\{0,1, z, \infty\} \rightarrow\{0,1, \phi(z), \infty\}$ be a permutation of $\{0,1, z, \infty\}$ followed by a Möbius transformation $z \mapsto$ $\phi(z)$ such that $\sigma$ preserves the cross ratio $(0,1 ; z, \infty)$, i.e. 
TABLE III. The census of $\phi^{4}$ periods. The numbers $Q$. are listed in Table I with definitions for the numbers $N$. in Table II. All known periods except for $P_{7,11}$ [23] can be calculated with [14]. See also [22] for definitions and explanations.

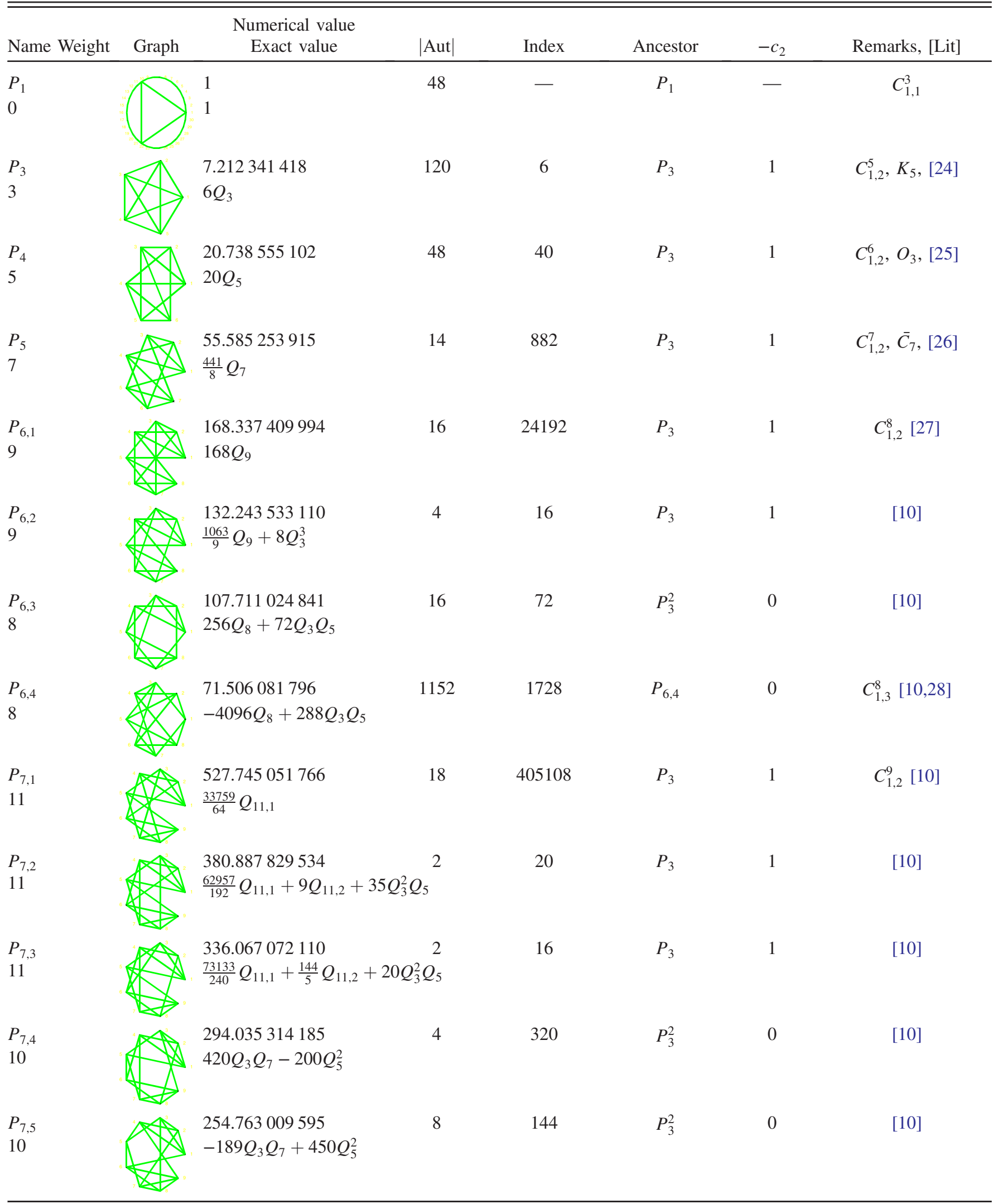


TABLE III. (Continued)

\begin{tabular}{|c|c|c|c|c|c|c|}
\hline Name Weight & Graph & $\begin{array}{l}\text { Numerical value } \\
\text { Exact value }\end{array}$ & Index & Ancestor & $-c_{2}$ & Remarks, [Lit] \\
\hline $\begin{array}{l}P_{7,6} \\
11\end{array}$ & & $\begin{array}{l}273.482574258 \\
\frac{14279}{64} Q_{11,1}-51 Q_{11,2}+35 Q_{3}^{2} Q_{5}\end{array}$ & 20 & $P_{3}$ & 1 & [10] \\
\hline $\begin{array}{l}P_{7,7} \\
10\end{array}$ & & $\begin{array}{l}294.035314185 \\
P_{7,4}\end{array}$ & 320 & $P_{3}^{2}$ & 0 & Fourier, twist \\
\hline $\begin{array}{l}P_{7,8} \\
11\end{array}$ & & $\begin{array}{l}183.032420030 \\
\frac{22383}{20} Q_{11,1}-\frac{4572}{5} Q_{11,2}+1792 Q_{3} Q_{8}\end{array}$ & $\begin{array}{r}16 \\
0 Q_{3}^{2} Q_{5}\end{array}$ & $P_{7,8}$ & $z_{2}$ & \\
\hline $\begin{array}{l}P_{7,9} \\
11\end{array}$ & & $\begin{array}{lc}216.919375587 & 12 \\
\frac{92943}{160} Q_{11,1}-\frac{3381}{20} Q_{11,2}+896 Q_{3} Q_{8}\end{array}$ & $Q_{3}^{2} Q_{5}^{3}$ & $P_{7,9}$ & $z_{2}$ & [29] \\
\hline $\begin{array}{l}P_{7,10} \\
10\end{array}$ & & $\begin{array}{l}254.763009595 \\
P_{7,5}\end{array}$ & 144 & $P_{7,10}$ & 0 & $K_{3} \square K_{3}$, Fourier \\
\hline $\begin{array}{l}P_{7,11} \\
11\end{array}$ & & $\begin{array}{l}200.357566429 \\
Q_{11,3}\end{array}$ & $?$ & $P_{7,11}$ & $z_{3}$ & $C_{1,3}^{9},[23]$ \\
\hline $\begin{array}{l}P_{8,1} \\
13\end{array}$ & & $\begin{array}{l}1716.210576104 \\
1716 Q_{13,1}\end{array}$ & 2635776 & $P_{3}$ & 1 & $C_{1,2}^{10}$ \\
\hline $\begin{array}{l}P_{8,2} \\
13\end{array}$ & & $\begin{array}{lc}1145.592929599 & 2 \\
\frac{25147347}{22400} Q_{13,1}-\frac{16881}{1400} Q_{13,2}+\frac{459}{112} Q_{13}\end{array}$ & $\begin{array}{c}12 \\
\frac{305}{8} Q_{3}^{2} Q_{7}-\end{array}$ & ${ }_{3} Q_{5}^{2}$ & 1 & \\
\hline $\begin{array}{l}P_{8,3} \\
13\end{array}$ & & $\begin{array}{l}1105.107697390 \\
298 Q_{13,1}+56 Q_{13,2}-20 Q_{13,3}-28\end{array}$ & $\begin{array}{c}1280 \\
Q_{7}+800 Q\end{array}$ & $P_{3}$ & 1 & \\
\hline $\begin{array}{l}P_{8,4} \\
13\end{array}$ & & $\begin{array}{lc}966.830801986 & 1 \\
\frac{17124243}{22400} Q_{13,1}-\frac{19689}{1400} Q_{13,2}+\frac{1755}{112} Q_{13}\end{array}$ & $\frac{9}{8} Q_{3}^{2} Q_{7}+$ & $Q_{5}^{2} P_{3}$ & 1 & \\
\hline $\begin{array}{l}P_{8,5} \\
12\end{array}$ & & $\begin{array}{lc}844.512518603 & 4 \\
1536 Q_{12,1}-1280 Q_{12,2}+36 Q_{3} Q_{9}\end{array}$ & $\begin{array}{r}24 \\
9 \\
Q_{5} Q_{7}\end{array}$ & $P_{3}^{2}$ & 0 & \\
\hline $\begin{array}{l}P_{8,6} \\
13\end{array}$ & & $\begin{array}{l}904.280824357 \\
\frac{214841}{336} Q_{13,1}-\frac{423}{7} Q_{13,2}+\frac{705}{14} Q_{13,3}+\end{array}$ & ${ }_{3}^{2} Q_{7}^{32}$ & $P_{3}$ & 1 & \\
\hline $\begin{array}{l}P_{8,7} \\
13\end{array}$ & & $\begin{array}{l}847.646115639 \\
\frac{2061501}{2800} Q_{13,1}+\frac{13527}{175} Q_{13,2}-\frac{675}{14} Q_{13,3}\end{array}$ & 144 & $P_{3}$ & 1 & \\
\hline
\end{tabular}


TABLE III. (Continued)

\begin{tabular}{|c|c|c|c|c|c|c|c|}
\hline Name Weight & Graph & $\begin{array}{c}\text { Numerical value } \\
\text { Exact value }\end{array}$ & $\mid$ Aut $\mid$ & Index & Ancestor & $-c_{2}$ & Remarks, [Lit] \\
\hline $\begin{array}{l}P_{8,8} \\
13\end{array}$ & & $\begin{array}{l}847.646115639 \\
P_{8,7}\end{array}$ & 2 & 144 & $P_{3}$ & 1 & twist \\
\hline $\begin{array}{l}P_{8,9} \\
13\end{array}$ & & $\begin{array}{l}904.280824357 \\
P_{8,6}\end{array}$ & 2 & 32 & $P_{3}$ & 1 & twist \\
\hline $\begin{array}{l}P_{8,10} \\
12\end{array}$ & & $\begin{array}{l}735.764103468 \\
1536 Q_{12,1}-1280 Q_{12}\end{array}$ & $\begin{array}{c}2 \\
Q_{3} Q_{9}\end{array}$ & ${ }_{5}{ }^{72}$ & $P_{3}^{2}$ & 0 & \\
\hline $\begin{array}{l}P_{8,11} \\
12\end{array}$ & & $\begin{array}{l}805.347388507 \\
\frac{10240}{69} Q_{12,1}+\frac{81920}{69} Q_{12}\end{array}$ & $\begin{array}{c}4 \\
0 \\
-\end{array}$ & $\begin{array}{c}16 \\
Q_{3} Q_{9}+\end{array}$ & $\begin{array}{c}P_{3}^{2} \\
Q_{7}-12 Q\end{array}$ & 0 & \\
\hline $\begin{array}{l}P_{8,12} \\
12\end{array}$ & & $\begin{array}{l}688.898361296 \\
1024 Q_{12,2}-1008 Q_{3}\end{array}$ & $\begin{array}{c}2 \\
800 Q_{5}\end{array}$ & 288 & $P_{3}^{2}$ & 0 & \\
\hline $\begin{array}{l}P_{8,13} \\
13\end{array}$ & & $\begin{array}{l}742.977090366 \\
\frac{10087273}{9600} Q_{13,1}+\frac{8007}{200} Q\end{array}$ & $\begin{array}{c}1 \\
\frac{13}{6} Q_{13},\end{array}$ & $\begin{array}{c}4 \\
Q_{3}^{2} Q_{7}-\end{array}$ & ${ }_{3}^{2}{ }^{P_{3}}$ & 1 & \\
\hline $\begin{array}{l}P_{8,14} \\
13\end{array}$ & & $\begin{array}{l}749.818622995 \\
\frac{41038969}{67200} Q_{13,1}-\frac{30129}{1400} Q\end{array}$ & $\begin{array}{c}1 \\
\frac{1611}{112} Q_{1}\end{array}$ & $\begin{array}{c}4 \\
{ }_{3}^{2} \\
Q_{3}^{2} Q_{7}\end{array}$ & $Q_{3} Q_{5}^{2}$ & 1 & \\
\hline $\begin{array}{l}P_{8,15} \\
12\end{array}$ & & $\begin{array}{l}805.347388507 \\
P_{8,11}\end{array}$ & 2 & 16 & $P_{3}^{2}$ & 0 & twist \\
\hline $\begin{array}{l}P_{8,16} \\
11,10\end{array}$ & & $\begin{array}{l}633.438914549 \\
-\frac{31851}{5} Q_{11,1}+\frac{24336}{5} Q\end{array}$ & $\begin{array}{c}32 \\
10240\end{array}$ & $\begin{array}{c}576 \\
5040 Q\end{array}$ & $\begin{array}{c}P_{3}^{3} \\
3192 Q_{10}+\end{array}$ & $\begin{array}{c}0 \\
2_{3} Q_{7}\end{array}$ & $0 Q_{5}^{2}$ \\
\hline $\begin{array}{l}P_{8,17} \\
13\end{array}$ & & $\begin{array}{l}589.354510434 \\
\frac{15548993}{4800} Q_{13,1}-\frac{17313}{100} Q\end{array}$ & $\begin{array}{c}2 \\
\frac{267}{8} Q_{13}\end{array}$ & $\begin{array}{c}8 \\
2 Q_{3} Q_{10}\end{array}$ & $\begin{array}{c}P_{3} \\
4 Q_{5} Q_{8}-\underline{8}\end{array}$ & $\begin{array}{c}1 \\
7-1\end{array}$ & \\
\hline $\begin{array}{l}P_{8,18} \\
12\end{array}$ & & $\begin{array}{l}641.723358297 \\
727 Q_{3} Q_{9}-\frac{735}{2} Q_{5} Q_{7}\end{array}$ & 2 & 48 & $P_{3}^{2}$ & 0 & \\
\hline $\begin{array}{l}P_{8,19} \\
12\end{array}$ & & $\begin{array}{l}598.617690750 \\
\frac{10240}{69} Q_{12,1}+\frac{81920}{69} Q_{12}\end{array}$ & $\begin{array}{c}4 \\
Q_{12,3}\end{array}$ & $\begin{array}{c}32 \\
Q_{3} Q_{9}+\end{array}$ & $\begin{array}{c}P_{3}^{2} \\
Q_{5} Q_{7}-84\end{array}$ & 0 & \\
\hline $\begin{array}{l}P_{8,20} \\
13\end{array}$ & & $\begin{array}{l}641.346699620 \\
\frac{4375463}{44800} Q_{13,1}+\frac{383001}{2800}\end{array}$ & $\begin{array}{c}1 \\
23607 \\
224\end{array}$ & $\begin{array}{c}6 \\
56 Q_{3} Q\end{array}$ & $\begin{array}{c}P_{3} \\
6 Q_{5} Q_{8}-1\end{array}$ & $\begin{array}{c}1 \\
Q_{7}+\end{array}$ & \\
\hline
\end{tabular}


TABLE III. (Continued)

\begin{tabular}{|c|c|c|c|c|c|c|c|}
\hline Name Weight & Graph & $\begin{array}{c}\text { Numerical value } \\
\text { Exact value }\end{array}$ & $\mid$ Aut $\mid$ & Index & Ancestor & $-c_{2}$ & Remarks, [Lit] \\
\hline $\begin{array}{l}P_{8,21} \\
13\end{array}$ & & $\begin{array}{l}742.977090366 \\
P_{8,13}\end{array}$ & 2 & 4 & $P_{3}$ & 1 & Fourier, twist \\
\hline $\begin{array}{l}P_{8,22} \\
12\end{array}$ & & $\begin{array}{l}735.764103468 \\
P_{8,10}\end{array}$ & 4 & 72 & $P_{3}^{2}$ & 0 & twist \\
\hline $\begin{array}{l}P_{8,23} \\
13\end{array}$ & & $\begin{array}{l}589.354510434 \\
P_{8,17}\end{array}$ & 2 & 8 & $P_{3}$ & 1 & twist \\
\hline $\begin{array}{l}P_{8,24} \\
13\end{array}$ & & $\begin{array}{l}414.873975722 \\
-\frac{40309047}{1400} Q_{13,1}-\frac{3536}{350}\end{array}$ & $\begin{aligned} & 8 \\
+ & \frac{4805}{28}\end{aligned}$ & $\begin{array}{c}144 \\
-17920\end{array}$ & $\begin{array}{c}P_{7,8} \\
-19840 Q\end{array}$ & $\begin{array}{c}z_{2} \\
\frac{17577}{2}\end{array}$ & $+10800 Q_{3} Q_{5}^{2}$ \\
\hline $\begin{array}{l}P_{8,25} \\
12\end{array}$ & & $\begin{array}{l}641.723358297 \\
P_{8,18}\end{array}$ & 4 & 48 & $P_{3}^{2}$ & 0 & Fourier, twist \\
\hline $\begin{array}{l}P_{8,26} \\
13\end{array}$ & & $\begin{array}{l}500.445152216 \\
\frac{25114323}{22400} Q_{13,1}-\frac{113979}{1400}\end{array}$ & $\begin{array}{c}4 \\
-\frac{4443}{112} Q\end{array}$ & $\begin{array}{c}6 \\
96 Q_{3} Q_{1}\end{array}$ & $\begin{array}{c}P_{7,9} \\
84 Q_{5} Q_{8}+\end{array}$ & ${ }_{3}^{z_{2}} Q_{7}$ & ${ }_{3} Q_{5}^{2}$ \\
\hline $\begin{array}{l}P_{8,27} \\
12\end{array}$ & & $\begin{array}{l}598.617690750 \\
P_{8,19}\end{array}$ & 4 & 32 & $P_{7,10}$ & 0 & Fourier \\
\hline $\begin{array}{l}P_{8,28} \\
13\end{array}$ & & $\begin{array}{l}500.445152216 \\
P_{8,26}\end{array}$ & 4 & 6 & $P_{7,9}$ & $z_{2}$ & twist \\
\hline $\begin{array}{l}P_{8,29} \\
13\end{array}$ & & $\begin{array}{l}553.273794612 \\
\frac{78907643}{89600} Q_{13,1}-\frac{306689}{5600}\end{array}$ & $\begin{array}{c}2 \\
-\frac{16987}{448}\end{array}$ & $\frac{1}{\frac{10129}{32}} Q_{3}^{2}$ & $\begin{array}{r}P_{7,9} \\
\frac{275}{4} Q_{3} Q_{5}^{2}\end{array}$ & $z_{2}$ & \\
\hline $\begin{array}{l}P_{8,30} \\
13\end{array}$ & & $\begin{array}{l}\approx 505.5 \\
?\end{array}$ & 2 & $?$ & $P_{7,11}$ & $z_{3}$ & {$[30]$} \\
\hline $\begin{array}{l}P_{8,31} \\
13\end{array}$ & & $\begin{array}{l}460.088538246 \\
\frac{67363763}{5600} Q_{13,1}-\frac{36487}{175}\end{array}$ & $\begin{array}{c}4 \\
\frac{1913}{7} Q_{1}\end{array}$ & $\begin{array}{c}8 \\
92 Q_{3} Q\end{array}$ & $\begin{array}{c}P_{7,8} \\
36 Q_{5} Q_{8}+\end{array}$ & $Q_{7}^{z_{2}}$ & ${ }_{3} Q_{5}^{2}$ \\
\hline $\begin{array}{l}P_{8,32} \\
12\end{array}$ & & $\begin{array}{l}470.720125534 \\
-\frac{81920}{23} Q_{12,1}-\frac{655360}{23}\end{array}$ & $\begin{array}{c}16 \\
\frac{20480}{23} Q\end{array}$ & $\begin{array}{l}17280 \\
\frac{760}{23} Q_{3} Q\end{array}$ & $\begin{array}{r}P_{8,32} \\
\frac{60}{3} Q_{5} Q_{7}\end{array}$ & 0 & \\
\hline $\begin{array}{l}P_{8,33} \\
13\end{array}$ & & $\begin{array}{l}468.038498992 \\
Q_{13,4}\end{array}$ & 2 & $?$ & $P_{8,33}$ & $z_{3}$ & \\
\hline
\end{tabular}


TABLE III. (Continued)

\begin{tabular}{|c|c|c|c|c|c|c|c|}
\hline Name Weight & Graph & $\begin{array}{c}\text { Numerical value } \\
\text { Exact value }\end{array}$ & |Aut $\mid$ & Index & Ancestor & $-c_{2}$ & Remarks, [Lit] \\
\hline $\begin{array}{l}P_{8,34} \\
12\end{array}$ & & $\begin{array}{l}470.720125534 \\
P_{8,32}\end{array}$ & 16 & 17280 & $P_{8,34}$ & 0 & twist \\
\hline $\begin{array}{l}P_{8,35} \\
13\end{array}$ & & $\begin{array}{l}\approx 460.2 \\
P_{8,31} ?\end{array}$ & 16 & $?$ & $P_{8,35}$ & $z_{2}$ & Hepp, [30] \\
\hline $\begin{array}{l}P_{8,36} \\
13\end{array}$ & & $\begin{array}{l}\approx 505.5 \\
P_{8,30} ?\end{array}$ & 10 & $?$ & $P_{8,36}$ & $z_{3}$ & Hepp, [30] \\
\hline $\begin{array}{l}P_{8,37} \\
?\end{array}$ & & $\begin{array}{l}\approx 422.9 \\
?\end{array}$ & 2 & $?$ & $P_{8,37}$ & $(3,7)$ & [30] \\
\hline $\begin{array}{l}P_{8,38} \\
?\end{array}$ & & $\begin{array}{l}\approx 386.6 \\
?\end{array}$ & 4 & $?$ & $P_{8,38}$ & $(4,5)$ & {$[30]$} \\
\hline $\begin{array}{l}P_{8,39} \\
?\end{array}$ & & $\begin{array}{l}\approx 384.2 \\
?\end{array}$ & 8 & $?$ & $P_{8,39}$ & $(3,8)$ & {$[30]$} \\
\hline $\begin{array}{l}P_{8,40} \\
13\end{array}$ & & $\begin{array}{l}\approx 312.1 \\
?\end{array}$ & 320 & $?$ & $P_{8,40}$ & $z_{4}$ & $C_{1,4}^{10},[30]$ \\
\hline $\begin{array}{l}P_{8,41} \\
?\end{array}$ & & $\begin{array}{l}\approx 323.3 \\
?\end{array}$ & 240 & $?$ & $P_{8,41}$ & $(6,3)$ & $C_{1,3}^{10},[30]$ \\
\hline
\end{tabular}

$$
\frac{-z}{1-z}=\frac{(\sigma(0)-\sigma(z))(\sigma(1)-\sigma(\infty))}{(\sigma(1)-\sigma(z))(\sigma(0)-\sigma(\infty))}
$$

Then $f_{\bar{G}}^{(\lambda)}=f_{\sigma(\bar{G})}^{(\lambda)}$, where the Möbius transformation in the label of $\sigma(\bar{G})$ acts on the argument of the graphical function. In particular, $f_{\bar{G}}^{(\lambda)}$ is invariant under double transpositions of external labels.

Because edges between external vertices produce trivial factors, completed graphical functions with stripped off edges between external vertices are equivalence classes graphical functions of the same type. In four dimensions, all those graphical functions with edge weights one and at most seven vertices are known [14].

Example 4.2. The smallest nontrivial graphical function has four vertices and three edges (see Fig. 3). In four dimensions, this graphical function is given by the BlochWigner dilogarithm (see e.g. [44]).

$$
f_{G}^{(\lambda)}(z)=\frac{4 \mathrm{i} D(z)}{z-\bar{z}}
$$

with

$$
D(z)=\operatorname{Im}\left(\operatorname{Li}_{2}(z)+\ln (1-z) \ln |z|\right)
$$

Theorem 4.1 and $f_{G}^{(\lambda)}(z)=f_{G}^{(\lambda)}(\bar{z})$ reflect the symmetries of $D$.
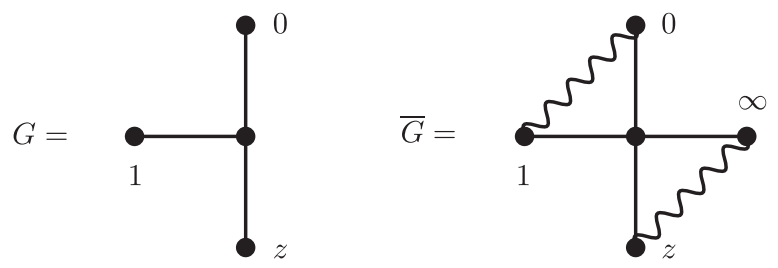

FIG. 3. A four dimensional graphical function with three edges and its completion. The wiggly lines refer to edge weights -1 . 


\section{B. Existence}

Existence of the graphical function $f_{G}^{(\lambda)}$ is best formulated in terms of the completion $\bar{G}$.

Theorem 4.3. (Lemma 3.19 in [9]). The graphical function $f_{\bar{G}}^{(\lambda)}$ exists if and only if for any vertex subset $V \subset \mathcal{V}(\bar{G})$ with at least two vertices and at most one external vertex the following inequality holds,

$$
(d-2) N_{g}<d(|V|-1),
$$

where $N_{g}$ is the sum of edge weights in the induced subgraph $g$ of $V$ in $\bar{G}$ (i.e. $g$ has all the edges of $\bar{G}$ that have both vertices in $V$ ).

In Example 4.2, we only have the case $|V|=2$ and $N_{g}=1$, so that $f_{G}^{(\lambda)}(z)$ exists. In $d$ dimensions the edge $\{z, \infty\}$ has weight $(6-d) /(d-2)$ so that (13) implies that the graphical functions of Example 4.2 exists in any dimension greater than three.

\section{General properties of graphical functions}

Graphical functions should be considered as functions on $\bar{C}=\mathbb{C} \cup\{\infty\}$. They have the following general properties:

Theorem 4.4. Let $G$ be a graph such that the graphical function $f_{G}^{(\lambda)}$ exists. (G1)

$$
f_{G}^{(\lambda)}(z)=f_{G}^{(\lambda)}(\bar{z})
$$

(G2) $f_{G}^{(\lambda)}$ is a positive single-valued real analytic function on $\bar{C} \backslash\{0,1, \infty\}$.

(G3) The radius of convergence of the real analytic expansion of $f_{G}^{(\lambda)}$ at $z_{0} \in \bar{C} \backslash\{0,1, \infty\}$ is the distance from $z_{0}$ to the nearest singularity of $f_{G}^{(\lambda)}$.

Let $\nu_{z}^{>}\left(\nu_{z}^{<}\right)$be the sum of positive (negative) weights of edges adjacent to $z$. Let the dimension $d=2 \lambda+2$ be even. Then, if $z_{0} \in\{0,1\}$, we have for $\left|z-z_{0}\right|<1$ and coefficients $c_{\ell, m, n}\left(z_{0}\right) \in \mathbb{C}$ :

$$
\begin{aligned}
f_{G}^{(\lambda)}(z)= & \sum_{\ell=0}^{V^{\mathrm{int}}} \sum_{m=M_{z_{0}}}^{\infty} \sum_{n=N_{z_{0}}}^{\infty} c_{\ell, m, n}\left(z_{0}\right) \\
& \times \log ^{\ell}\left[\left(z-z_{0}\right)\left(\bar{z}-\overline{z_{0}}\right)\right]\left(z-z_{0}\right)^{m}\left(\bar{z}-\overline{z_{0}}\right)^{n},
\end{aligned}
$$

where

$$
M_{z_{0}}, \quad N_{z_{0}}>-\lambda \nu_{z}^{>} .
$$

If $z_{0}=\infty$ we have for $|z|>1$ and coefficients $c_{\ell, m, n}(\infty) \in \mathbb{C}:$

$$
f_{G}^{(\lambda)}(z)=\sum_{\ell=0}^{V^{\mathrm{int}}} \sum_{m=-\infty}^{M_{\infty}} \sum_{n=-\infty}^{N_{\infty}} c_{\ell, m, n}(\infty) \log { }^{\ell}(z \bar{z}) z^{m} \bar{z}^{n},
$$

where

$$
M_{\infty}, \quad N_{\infty}<-\lambda \nu_{z}^{<}
$$

Property (G1) is immediate by symmetry. It also follows from the parametric representation of graphical functions [8]. Property (G2) is proved in [8] while (G3) will be handled in [45]. Conjecture 4.12 gives additional information on the leading terms of the above expansions. In the case of odd dimensions, there exist expansions at $0,1, \infty$ which are similar to (15) and (16) with a square root $\sqrt{\left(z-z_{0}\right)\left(\bar{z}-\bar{z}_{0}\right)}[45]$.

\section{Appending edges}

Edges between external vertices give factors in a graphical function. In the case that an edge is appended to the vertex $z$ creating a new vertex $z$ (see Fig. 4), the graphical functions are related by a differential equation $[9,46]$.

Lemma 4.5. In the setup of Fig. 4, we have

$$
\begin{aligned}
& \left(-\frac{1}{(z-\bar{z})} \partial_{z} \partial_{\bar{z}}(z-\bar{z})+\frac{\lambda-1}{z-\bar{z}}\left(\partial_{z}-\partial_{\bar{z}}\right)\right) f_{G_{1}}^{(\lambda)}(z) \\
& \quad=\frac{1}{\Gamma(\lambda)} f_{G}^{(\lambda)}(z)
\end{aligned}
$$

where $\Gamma(\lambda)=\int_{0}^{\infty} x^{\lambda-1} \exp (-x) \mathrm{d} x$ is the gamma function.

The differential equation is particularly simple in $d=4$ dimensions $(\lambda=1)$. In this case, we (uniquely) obtain the graphical function of $G_{1}$ by single-valued integration with respect to $z$ and $\bar{z}$ (see [9]).

Lemma 4.6. Let $\lambda=1$. The differential operator on the left-hand side of (17) has trivial kernel in the space of functions with general properties (G1)-(G3).

Proof.-Assume $f$ with properties (G1)-(G3) is in the kernel of the differential operator for $\lambda=1$. Because of (G2), $g(z)=\partial_{z}(z-\bar{z}) f(z)$ is meromorphic. We use (15) with $N_{0}, M_{0}, N_{1}, M_{1} \geq 0$ (because $\nu_{z}^{>}=1$ in $G_{1}$ ) and conclude that $g$ is holomorphic on $\mathbb{C}$. From (16) with $N_{\infty}, M_{\infty} \leq-1$ we get $g(\infty)=0$, hence, by Liouville's

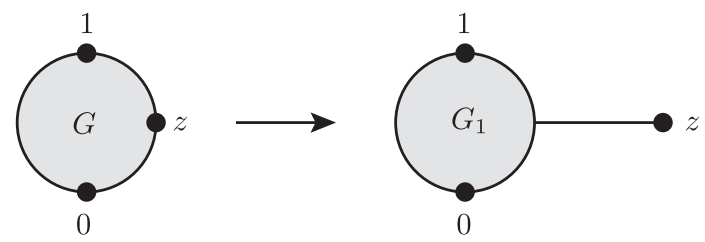

FIG. 4. Appending an edge to the vertex $z$ in $G$ gives $G_{1}$. 
theorem, $g=0$. Therefore $f=h(\bar{z}) /(z-\bar{z})$ for some antiholomorphic function $h$. With (G1) we obtain $h=0$.

Beginning with the empty graphical function we can construct many graphical functions by appending edges (see $[9,11])$. A particularly simple class of such graphical functions is handled by the following theorem.

Theorem 4.7. Let $G$ be a graph with external vertex width four (constructible in [9]), i.e. $G$ can be constructed from the empty graph by adding edges between external vertices, permuting external vertices, and appending edges. If $G$ has no edges between external vertices then we obtain in $d=4$ dimensions,

$$
f_{G}^{(1)}(z)=P(z) /(z-\bar{z}),
$$

where $P$ is a single-valued multiple polylogarithm [47] of weight $2 V^{\text {int }}(G)$.

The proof of the theorem will be in [45]. Note that the theorem is consistent with Example 4.2.

In $d=4-\epsilon$ dimensions, edges can still be appended if one expands $f_{G}^{(\lambda)}$ into a Laurent series in $\epsilon$ to a given order. The procedure, however, is more subtle, see Sec. IV D 8.

\section{E. Identities}

There exist many identities for graphical functions. A Fourier-identity relating planar duals was proved in [8]. Like in the case of periods there also exists a twist identity. Some complicated graphical functions can be calculated by a Gegenbauer technique [9,45]. All known identities are included in HyperlogProcedures which (among other things) can calculate many graphical functions [14]. If a graphical function is inaccessible to all of these methods then sometimes it can still be calculated by parametric integration, due to F. Brown $[48,49]$ and E. Panzer $[23,50]$.

In [51], B. Basso and L. J. Dixon provide an intriguingly simple formula for graphical functions of the $(m, n)$ 'fishnet' topology. The result is conjectured from methods and properties of $N=4$ supersymmetric Yang-Mills theory. For $m, n>1$ the fishnet topology is inaccessible to the tools presented here. This indicates that much more powerful methods may exist for the calculation of graphical functions.

\section{F. From graphical functions to periods}

There exist several options to derive Feynman periods from graphical functions.
First, one can specify the variable $z$ in $f_{G}^{(\lambda)}(z)$ to 0,1 , or $\infty$. In the case of the zig-zag graphs depicted in Fig. 5, this method leads to a proof of a conjecture by D. Broadhurst and D. Kreimer in 1995 [10].

Theorem 4.8. (F. Brown, O. Schnetz, [11]). The period of the graph $Z_{n}$ is given by

$P\left(Z_{n}\right)=4 \frac{(2 n-2) !}{n !(n-1) !}\left(1-\frac{1-(-1)^{n}}{2^{2 n-3}}\right) \zeta(2 n-3)$.

Second, in integer dimensions $d \geq 3$ one can integrate $f_{G}^{(\lambda)}(z)$ over the external variable $z$ [9]. This effectively makes $z$ an internal variable. For a general graph in integer dimensions this is the best method to calculate Feynman periods. It is used in [14].

Third, in the case of dimensional regularization we need to treat the dimension $d$ as a parameter and expand periods in $4-\epsilon$ dimensions at $\epsilon=0$. Using the second method one obtains an integration measure of $(z-\bar{z})^{2-\epsilon}$ which does not expand in $\epsilon$ into GSVHs (see Sec. IV D 7). One can resort to the following procedure to integrate over $z$ : Add an edge of weight -1 between the external vertices 0 and $z$. Then, append an edge to the vertex $z$ creating a new vertex $z$. Set $z=0$ so that the newly appended edge cancels the previously added edge of weight -1 . This integrates over $z$ in any possibly non-integer dimension $d$. This method is used for perturbative calculations in dimensionally regularized $\phi^{4}$ theory (see Sec. IV D 9) [14]. It is also possible to devise a more direct method to perform $4-\epsilon$ dimensional integrations (private communication with E. Panzer). However, this is conceptually more demanding and in practice the method that appends an edge works quite well.

\section{G. Generalized single-valued hyperlogarithms}

Many graphical functions can be expressed in terms of iterated integrals [52]. Although, by (G2) of Sec. IV D, graphical functions have only singularities at 0,1 , and $\infty$ it turns out that single-valued multiple polylogarithms [47] (i.e. letters 0 and 1 in the iterated integrals) are too restrictive. Let us consider the following example (which shows the same mechanism although it is not a graphical function).

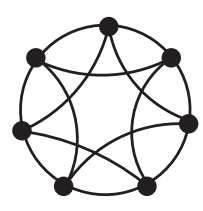

$Z_{5}$

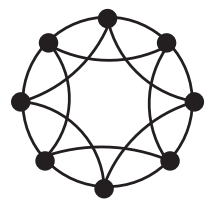

$Z_{6}$

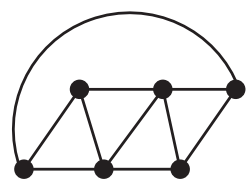

$Z_{5}$

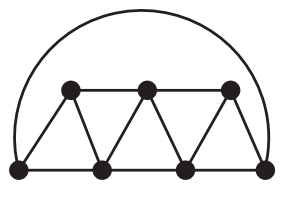

$Z_{6}$

FIG. 5. Completed $\left(\overline{Z_{\bullet}}\right)$ and uncompleted (Z.) zig-zag graphs with five and six loops. 
Example 4.9. let $r>0$. Define

$$
f(z)=\frac{\log (z \bar{z} / r)}{z-r / \bar{z}}
$$

Then $f$ is real analytic on $\bar{C} \backslash\{0, \infty\}$ because the zero locus $z=r / \bar{z}$ in the denominator is canceled by the numerator. By general principles, there should exist a single-valued primitive of $f$ which also is real analytic on $\bar{C} \backslash\{0, \infty\}$. By single-valuedness the primitive is determined up to a rational function in $\bar{z}$. It is unique in the space of hyperlogarithms which vanish at 0 .

We generalize the above example in the sense that we consider bilinear denominators in $z$ and $\bar{z}$ (i.e. $a+b z+c \bar{z}+d z \bar{z}$, with $a, b, c, d \in \mathbb{C})$. We require that non pointlike zero loci in the denominator are canceled by the numerator. This leads to functions with pointlike singularities, so that single-valuedness makes sense. We can construct single-valued primitives of single-valued functions leading to the space of generalized single-valued hyperlogarithms (GSVHs).

There also exist GSVHs with no singularities on the complex plane. A trivial example of this type is $\log (1+$ $z \bar{z} / r)$ for $r>0$. In the context of graphical functions, however, we have GSVHs of the type in Example 4.9. A QFT example of weight three is the single-valued primitive of $4 \mathrm{i} D(z) /(z-\bar{z})$ where $D$ is the Bloch-Wigner dilogarithm (12) (see also [53]).

Let $\mathcal{G}$ be the space of GSVHs. Upon differentiation with respect to $z$ and $\bar{z}$ we obtain the spaces $\partial_{z} \mathcal{G}, \partial_{\bar{z}} \mathcal{G}, \partial_{\bar{z}} \partial_{z} \mathcal{G}$. For example, $f$ in (20) is in $\partial_{z} \mathcal{G}$. We would like to construct an algorithm for single-valued integration in $\partial_{z} \mathcal{G}$.

Functions in $\partial_{z} \mathcal{G}$ have expansions (15) and (16) for general $z_{0} \in \bar{C}$. The holomorphic residue $\operatorname{res}_{z_{0}}$ is the coefficient $c_{0,-1,0}\left(z_{0}\right)$, whereas the anti-holomorphic residue rēs $z_{z_{0}}$ is the coefficient $c_{0,0,-1}\left(z_{0}\right)$. let $\pi_{0}\left(\bar{\pi}_{0}\right)$ be the projection onto the (anti-)residue free part,

$$
\begin{aligned}
& \pi_{0}: \partial_{z} \mathcal{G} \rightarrow \partial_{z} \mathcal{G}, f(z) \mapsto f(z)-\sum_{z_{0} \in \mathbb{C}} \frac{\operatorname{res}_{z_{0}}(f)}{z-z_{0}}, \\
& \bar{\pi}_{0}: \partial_{\bar{z}} \mathcal{G} \rightarrow \partial_{\bar{z}} \mathcal{G}, \quad f(z) \mapsto f(z)-\sum_{z_{0} \in \mathbb{C}} \frac{\operatorname{res}_{z_{0}}(f)}{\bar{z}-\overline{z_{0}}} .
\end{aligned}
$$

An efficient method to obtain single-valued primitives relies on the commutative hexagon in Fig. 6, where $\int_{\mathrm{sv}}$ stands for single-valued integration.

Theorem 4.10. The diagram in Fig. 6 commutes.

The proof of the theorem will be in [13]. By virtue of Fig. 6, we can express a single-valued primitive with respect to $z$ also as a single-valued primitive with respect to $\bar{z}$. Because single-valued integration in $z$ (resp. $\bar{z}$ ) equals ordinary integration up to an anti-holomorphic (resp. holomorphic) function, knowing both integrands determines the single-valued primitive up to a constant (which

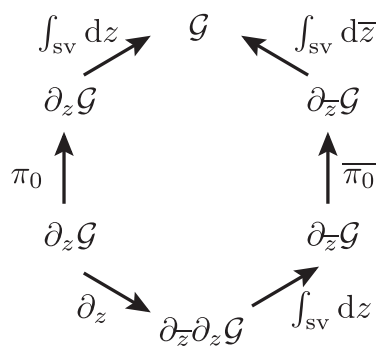

FIG. 6. The inductive construction of GSVHs by a commutative hexagon.

is fixed by the condition that the single-valued primitive vanishes at $z=0$ ). Using integration by parts at the bottom right arrow in Fig. 6, we can reduce single-valued integration to lower weights.

If $f \in \partial_{z} \mathcal{G}$ has weight 0 then the integration from $\partial_{\bar{z}} \partial_{z} \mathcal{G}$ to $\partial_{\bar{z}} \mathcal{G}$ is purely rational. The single-valued integration of the residues (which has to be done separately) is trivial: The single-valued primitive of $1 /(z-c)$ is $\log [(z-c)(\bar{z}-\bar{c})]$ for any $c \in \mathbb{C}$.

Example 4.11. (Example 4.9 continued). In terms of iterated integrals (writing from right to left), we obtain for the numerator of the integrand

$$
\log (z \bar{z} / r)=I(z, 0,0)+I(\bar{z}, 0,0)-I(r, 0,0) .
$$

The single-valued primitive of $f$ has the general form (note that $f$ is residue-free)

$$
\begin{aligned}
\int_{\mathrm{sv}} f(z) \mathrm{d} z= & I(z, r / \bar{z}, 0,0) \\
& +I(z, r / \bar{z}, 0)[I(\bar{z}, 0,0)-I(r, 0,0)] \\
& +g(\bar{z})
\end{aligned}
$$

for some anti-holomorphic $g$. Differentiation with respect to $\bar{z}$ yields

$$
\partial_{\bar{z}} f(z)=\frac{1}{z \bar{z}-r}-r \frac{\log (z \bar{z} / r)}{(z \bar{z}-r)^{2}}
$$

Using integration by parts on the second term, we find

$$
\int_{\mathrm{sv}}-r \frac{\log (z \bar{z} / r)}{(z \bar{z}-r)^{2}} \mathrm{~d} z=\frac{r \log (z \bar{z} / r)}{\bar{z}(z \bar{z}-r)}-\int_{\mathrm{sv}} \frac{r}{z \bar{z}(z \bar{z}-r)} \mathrm{d} z .
$$

Adding the two terms which remain to be integrated, the factor $(z \bar{z}-r)$ cancels (it has to) and we obtain

$$
\int_{\mathrm{sv}} \partial_{\bar{z}} f(z) \mathrm{d} z=\frac{r \log (z \bar{z} / r)}{\bar{z}(z \bar{z}-r)}+\frac{\log (z \bar{z})}{\bar{z}} .
$$

In fact, there is an ambiguity in form of a rational function in $\bar{z}$. However, because the result has to be in $\partial_{\bar{z}} \mathcal{G}$, the 
ambiguity can only be an anti-residue and it is removed by the projection $\overline{\pi_{0}}$. The above expression has an anti-residue at $z=0$ with value $\log (r)$. Subtraction yields

$$
\bar{\pi}_{0} \int_{\mathrm{sv}} \partial_{\bar{z}} f(z) \mathrm{d} z=\frac{\log (z \bar{z} / r)}{\bar{z}-r / z} .
$$

Using the commutative hexagon we obtain by integration with respect to $\bar{z}$,

$$
\begin{aligned}
\int_{\mathrm{sv}} f(z) \mathrm{d} z= & I(\bar{z}, r / z, 0,0) \\
& +I(\bar{z}, r / z, 0)[I(z, 0,0)-I(r, 0,0)] \\
& +h(z)
\end{aligned}
$$

for some holomorphic function $h$. If we write (22) as hyperlogarithms in $z$ with coefficients which are hyperlogarithms in $\bar{z}$ we get (21) with $h(z)$ instead of $g(\bar{z})$. (Alternatively we may treat $z$ and $\bar{z}$ as independent variables in (22) and consider the limit $z \rightarrow 0$.) We conclude that in this example $h(z)=g(\bar{z})$ is a constant. This constant is zero because the single-valued integral is required to vanish at $z=0$.

At four dimensions some (few) graphical functions exist which can be expressed in terms of ordinary single-valued multiple polylogarithms (see e.g. [11]). A large majority of graphical functions which can be expressed in terms of iterated integrals are GSVHs which are not single-valued multiple polylogarithms. In $4-\epsilon$ "dimensions," every nontrivial graphical function expands in $\epsilon$ with coefficients which are not single-valued multiple polylogarithms (but often GSVHs).

\section{H. 4- $\epsilon$ dimensions}

We can use the parametric representation of graphical functions [8] to define graphical function for non-integer $d$. Using $4-\epsilon$ 'dimensions' regularizes graphical functions: Graphical functions that diverge in 4 dimensions may (and generically do) exist in $4-\epsilon$ dimensions.

Although general properties (G1) and (G2) in Theorem 4.4 remain valid, graphical functions in noninteger dimensions can hardly be calculated in terms of known functions. However, in QFT it suffices to know their Laurent expansions at $\epsilon=0$ to some (small) order in $\epsilon$. For the Laurent coefficients, (G3) holds and often they can be expressed in terms of GSVHs.

The main tool for constructing these coefficients is again appending edges (see Sec. IV D 4). Equation (17) can be solved iteratively in powers of $\epsilon$. However, in this approach we cannot directly use Lemma 4.6 to avoid the kernel of the differential operator. We first need to subtract poles at $z=z_{0}, z_{0} \in\{0,1\}$, which are of order four (or higher) in $\left|z-z_{0}\right|$. (Using completion an analogous subtraction is necessary at $z_{0}=\infty$.) For these singular contributions we need exact results; it is not sufficient to know them to a limited order in $\epsilon$. In a renormalizable QFT, we only have to deal with "logarithmic" singularities; i.e. the case of poles of order four suffices. This leading-order asymptotic behavior of graphical functions is obtained by the following conjecture.

Conjecture 4.12. Let $G$ be a graph with $\mathcal{V}^{\text {int }}$ internal and $\mathcal{V}^{\text {ext }}=\{0,1, z\}$ external vertices such that the graphical function $f_{G}^{(\lambda)}$ exists. Let $G[V]$ be the subgraph of $G$ which is induced by $V$, i.e. the subgraph which contains the vertices $V$ and all edges of $G$ with both vertices in $V$. Further let $z_{0} \in$ $\{0,1\}$ and $G\left[V=z_{0}\right]$ be the graph $G / G[V]$ where one identifies all vertices in $V$ with the vertex $z_{0}$. Then (see Fig. 7). we obtain the asymptotic expansions at $z=z_{0}$ by

$$
f_{G}^{(\lambda)}(z)=\sum_{V \subseteq \mathcal{V}^{\text {int }}} f_{G\left[V \cup\left\{z_{0}, z\right\}\right]}^{(\lambda)}(z) f_{G\left[V \cup\left\{z_{0}, z\right\}=z_{0}\right]}^{(\lambda)}\left(1+O\left(\left|z-z_{0}\right|^{2}\right)\right)
$$

whenever the right-hand side exists. The asymptotic expansion at $z=\infty$ is given by

$$
f_{G}^{(\lambda)}(z)=\sum_{V \subseteq \mathcal{V}^{\text {int }}} f_{G[V \cup\{0,1\}]}^{(\lambda)} f_{G[V \cup\{0,1\}=0]}^{(\lambda)}(z)\left(1+O\left(|z|^{-2}\right)\right)
$$

whenever the right-hand side exists.

Note that on the right-hand side of the above equations one has graphs with two external vertices [see (2)]. The calculation of their functions amounts to calculating periods (see Sec. III) which is much simpler than the calculation of graphical functions. Equations (23) and (24) are formally obtained by rescaling some internal variables $x_{i} \mapsto x_{i}|z|$ followed by a naive expansion in the integrand.

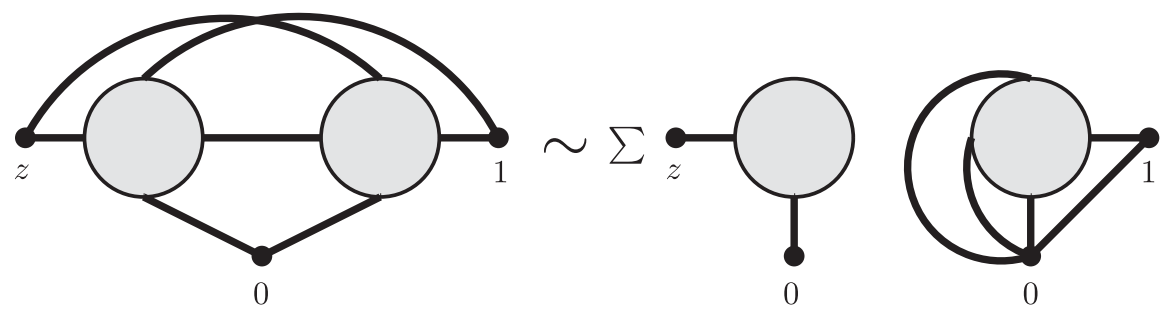

FIG. 7. The asymptotic expansion of graphical functions at $z=0$. The bold lines stand for sets of edges. 
The sum is over all possible ways to do this. In (rare) situations, the right-hand sides may fail to exist (although working in $4-\epsilon$ dimensions). In these cases, we have no result for the asymptotic expansion of the left-hand side.

In integer dimensions, Conjecture 4.12 is an analog to a power-counting result by S. Weinberg from 1960 [54]. In the mathematical literature, one uses the equivalent technique of blowing up singularities (see e.g. [55] in the QFT context). Here, however, we use $4-\epsilon$ dimensions so that the methods so not directly apply in position space. We thuse leave the result as a well tested conjecture.

Up to four loops appending edges and permuting external vertices suffices to calculate graphical functions. In four loops, all encountered functions are GSVHs in the alphabet $0,1, \bar{z}$ (this holds to all orders in $\epsilon$ ). Because such GSVHs evaluate to MZVs this extends a results by B. A. Baikov and K. G. Chetyrkin (up to weight 7) [56] and by
R. N. Lee, A. V. Smirnov, and V. A. Smirnov (up to weight 12) [57] to arbitrary weight.

In addition to appending edges, there exist a variety of tools that allows one to calculate the $\epsilon$-expansions of graphical functions in $4-\epsilon$ dimensions to low orders in $\epsilon$ [58]. Note that linear reducibility [23] and the method presented here suggest that MZVs exhaust the number contents of $\epsilon$ expansions up to 6 loops and any order in $\epsilon$.

\section{I. $\beta, \gamma, \gamma_{m}$, and the self-energy in dimensionally regularized $\phi^{4}$ theory}

Most efficiently one calculates $\phi^{4}$ renormalization functions in the minimal subtraction scheme of dimensional regularization [18]. The seven loop $\beta$ function, anomalous dimension $\gamma$, and anomalous mass dimension $\gamma_{m}$ are (The Feynman graphs were generated with M. Borinsky's program feyngen [59].)

$$
\begin{aligned}
\beta= & \left(\frac{195654269}{23040}+\frac{15676169}{720} \zeta(3)-\frac{316009}{3840} \pi^{4}+\frac{18326039}{480} \zeta(5)-\frac{129631}{5040} \pi^{6}+\frac{516957}{20} \zeta(3)^{2}-\frac{4453}{60} \pi^{4} \zeta(3)\right. \\
& +\frac{1536173}{20} \zeta(7)-\frac{20425591}{1260000} \pi^{8}+116973 \zeta(3) \zeta(5)+\frac{947214}{25} \zeta(5,3)-\frac{1010}{63} \pi^{6} \zeta(3)+\frac{613}{5} \pi^{4} \zeta(5)+4176 \zeta(3)^{3} \\
& +\frac{547118}{3} \zeta(9)-\frac{45106}{43659} \pi^{10}-48 \pi^{4} \zeta(3)^{2}+\frac{84231}{2} \zeta(3) \zeta(7)-\frac{273030}{7} \zeta(5)^{2}+\frac{8460}{7} \zeta(7,3)-\frac{174}{25} \pi^{8} \zeta(3) \\
& +\frac{6227}{35} \pi^{6} \zeta(5)-\frac{56043}{25} \pi^{4} \zeta(7)-504387 \pi^{2} \zeta(9)+46845 \zeta(3)^{2} \zeta(5)+27216 \zeta(3) \zeta(5,3)-\frac{336258}{5} \zeta(5,3,3) \\
& \left.+\frac{52756839}{10} \zeta(11)+24 P_{7,11}\right) g^{8}+\left(-\frac{18841427}{11520}-\frac{779603}{240} \zeta(3)+\frac{5663}{480} \pi^{4}-\frac{63723}{10} \zeta(5)+\frac{6691}{1890} \pi^{6}-\frac{8678}{5} \zeta(3)^{2}\right. \\
& \left.+\frac{9}{5} \pi^{4} \zeta(3)-\frac{63627}{5} \zeta(7)+\frac{88181}{78750} \pi^{8}-4704 \zeta(3) \zeta(5)-\frac{51984}{25} \zeta(5,3)-768 \zeta(3)^{3}-\frac{46112}{3} \zeta(9)\right) g^{7} \\
& +\left(\frac{764621}{2304}+\frac{7965}{16} \zeta(3)-\frac{1189}{720} \pi^{4}+987 \zeta(5)-\frac{5}{14} \pi^{6}+45 \zeta(3)^{2}+1323 \zeta(7)\right) g^{6} \\
& +\left(-\frac{3499}{48}-78 \zeta(3)+\frac{1}{5} \pi^{4}-120 \zeta(5)\right) g^{5}+\left(\frac{145}{8}+12 \zeta(3)\right) g^{4}-\frac{17}{3} g^{3}+3 g^{2} \\
\approx & 474651 g^{8}-34776.1 g^{7}+2848.57 g^{6}-271.606 g^{5}+32.5497 g^{4}-5.66667 g^{3}+3 g^{2},
\end{aligned}
$$

where $P_{7,11}$ is given in (3). This confirms (and goes beyond) a recent six loop result by M. V. Kompaniets and E. Panzer [43]. Note that the final result for the five loop $\beta$ function was already obtained in 1993 by H. Kleinert, J. Neu, V. SchulteFrohlinde, K. G. Chetyrkin and S. A. Larin [60]. In this article, the authors also give a five-loop result for $\gamma_{m}$ and a four-loop result for the anomalous dimension $\gamma$.

$$
\begin{aligned}
\gamma= & \left(-\frac{214519}{5120}-\frac{52883}{1920} \zeta(3)-\frac{4247}{23040} \pi^{4}+\frac{8023}{320} \zeta(5)-\frac{71}{1080} \pi^{6}-\frac{523}{40} \zeta(3)^{2}\right. \\
& \left.-\frac{1}{20} \pi^{4} \zeta(3)+\frac{3573}{40} \zeta(7)-\frac{2063}{210000} \pi^{8}+27 \zeta(3) \zeta(5)+\frac{162}{25} \zeta(5,3)\right) g^{7} \\
& +\left(\frac{73667}{9216}+\frac{295}{192} \zeta(3)+\frac{73}{1920} \pi^{4}-\frac{37}{8} \zeta(5)+\frac{5}{756} \pi^{6}-\frac{1}{2} \zeta(3)^{2}\right) g^{6} \\
& +\left(-\frac{3709}{2304}+\frac{3}{16} \zeta(3)-\frac{1}{180} \pi^{4}\right) g^{5}+\frac{65}{192} g^{4}-\frac{1}{16} g^{3}+\frac{1}{12} g^{2} \\
\approx & -124.159 g^{7}+14.3840 g^{6}-1.92558 g^{5}+0.338542 g^{4}-0.0625 g^{3}+0.0833333 g^{2} .
\end{aligned}
$$


This confirms (and goes beyond) a recent six-loop calculation of D. V. Batkovich, K. G. Chetyrkin, and M. V. Kompaniets [61].

$$
\begin{aligned}
\gamma_{m}= & \left(-\frac{24838423}{13824}-\frac{2399489}{864} \zeta(3)+\frac{329}{960} \pi^{4}-\frac{25511}{24} \zeta(5)-\frac{1865}{1134} \pi^{6}-\frac{140153}{48} \zeta(3)^{2}-\frac{68}{45} \pi^{4} \zeta(3)+\frac{46625}{12} \zeta(7)\right. \\
& -\frac{83003}{378000} \pi^{8}-4519 \zeta(3) \zeta(5)-\frac{6147}{5} \zeta(5,3)-\frac{412}{189} \pi^{6} \zeta(3)+\frac{167}{30} \pi^{4} \zeta(5)+424 \zeta(3)^{3}+\frac{60289}{12} \zeta(9) \\
& \left.-\frac{45106}{654885} \pi^{10}-\frac{16}{5} \pi^{4} \zeta(3)^{2}+\frac{777}{2} \zeta(3) \zeta(7)+\frac{31778}{7} \zeta(5)^{2}+\frac{564}{7} \zeta(7,3)\right) g^{7} \\
& +\left(\frac{7915913}{23040}+\frac{472891}{1440} \zeta(3)+\frac{113}{192} \pi^{4}+\frac{4019}{40} \zeta(5)+\frac{163}{540} \pi^{6}+\frac{446}{5} \zeta(3)^{2}+\frac{3}{5} \pi^{4} \zeta(3)-\frac{4629}{20} \zeta(7)+\frac{2063}{35000} \pi^{8}\right. \\
& \left.-288 \zeta(3) \zeta(5)-\frac{972}{25} \zeta(5,3)\right) g^{6}+\left(-\frac{158849}{2304}-\frac{1519}{48} \zeta(3)-\frac{13}{72} \pi^{4}-\zeta(5)-\frac{5}{126} \pi^{6}+9 \zeta(3)^{2}\right) g^{5} \\
& +\left(\frac{477}{32}+\frac{3}{2} \zeta(3)+\frac{1}{30} \pi^{4}\right) g^{4}-\frac{7}{2} g^{3}+\frac{5}{6} g^{2}-g \\
\approx & -13759.8 g^{7}+1354.64 g^{6}-150.756 g^{5}+19.9563 g^{4}-3.5 g^{3}+0.833333 g^{2}-g .
\end{aligned}
$$

This confirms (and goes beyond) a recent six-loop result by M. V. Kompaniets and E. Panzer [43].

The six-loop self-energy $\Sigma$ is

$$
\begin{aligned}
\frac{\Sigma(p)}{p^{2}}= & {\left[-\frac{27}{2} L^{5}-\frac{3643}{24} L^{4}+\left(-\frac{648011}{864}-16 \zeta(3)\right) L^{3}+\left(-\frac{291187}{144}-82 \zeta(3)-\frac{1}{20} \pi^{4}-20 \zeta(5)\right) L^{2}\right.} \\
& +\left(-\frac{1699885}{576}-\frac{32953}{192} \zeta(3)-\frac{11}{48} \pi^{4}-\frac{211}{4} \zeta(5)-\frac{5}{378} \pi^{6}+\zeta(3)^{2}\right) L-\frac{33992153}{18432}-\frac{683389}{4608} \zeta(3)-\frac{18403}{69120} \pi^{4} \\
& \left.-\frac{8681}{192} \zeta(5)-\frac{359}{18144} \pi^{6}-\frac{83}{48} \zeta(3)^{2}+\frac{1}{360} \pi^{4} \zeta(3)+\frac{5}{6} \zeta(7)\right] g^{6}+\left[\frac{9}{2} L^{4}+\frac{1375}{36} L^{3}+\left(\frac{12935}{96}+2 \zeta(3)\right) L^{2}\right. \\
& \left.+\left(\frac{8353}{36}+5 \zeta(3)+\frac{1}{90} \pi^{4}\right) L+\frac{1874629}{11520}+\frac{6319}{1440} \zeta(3)+\frac{251}{14400} \pi^{4}-\frac{1}{5} \zeta(5)\right] g^{5} \\
& +\left[-\frac{3}{2} L^{3}-\frac{53}{6} L^{2}-\frac{1867}{96} L-\frac{2017}{128}+\frac{3}{32} \zeta(3)\right] g^{4}+\left[\frac{1}{2} L^{2}+\frac{7}{4} L+\frac{167}{96}\right] g^{3}+\left[-\frac{1}{6} L-\frac{13}{48}\right] g^{2},
\end{aligned}
$$

where

$$
L=\frac{1}{2} \log \left(\frac{4 \pi \Lambda^{2}}{\exp (C) p^{2}}\right),
$$

with the renormalization scale $\Lambda$ and Euler-Mascheroni constant $C=0.577 \ldots$. This result confirms an unpublished five loop result by $\mathrm{D}$. Broadhurst for the propagator $1 /\left(p^{2}-\Sigma(p)\right)$ at $L=0$ [62].

The analogous results for the $O(n)$-symmetric model have also been calculated. They are available in HyperlogProcedures [14]. With these results E. Panzer improved his QFT predictions for critical exponents in three dimensional statistical models (private communication, [43]).

While eight loop calculations of the anomalous dimensions $\gamma$ and $\gamma_{m}$ seem possible, an eight loop result for the $\beta$ function demands serious determination. For the time being the author is not pursuing eight loop calculations.

\section{J. The anomalous magnetic moment of the electron}

So far, all results were obtained in the framework of massless bosonic $\phi^{4}$ theory. How does the picture change for a physical gauge theory with massive fermions? An excellent test is the QED contribution to the anomalous magnetic moment of the electron $a_{e}$ where three orders in $\alpha / \pi$ are known [3] with a recent partial fourth-order result by S. Laporta [4].

In the $f$ alphabet for MZVs with extensions by all sixth roots of unity, we obtain-we use the letters $g^{6}$ to make the distinction to (3) which refers to a number subset which has no weight 1 letters $g_{1}^{6} \cong 2 \log 2$ and $\log 3$ (the letter $\log 3$ is absent in all known terms of $a_{e}$ ): 


$$
\begin{aligned}
a_{e}= & \frac{1}{2}\left(\frac{\alpha}{\pi}\right)+\left(\frac{197}{144}+\frac{1}{12} \pi^{2}+\frac{27}{32} g_{3}^{6}-\frac{1}{4} g_{1}^{6} \pi^{2}\right)\left(\frac{\alpha}{\pi}\right)^{2} \\
& +\left(\frac{28259}{5184}+\frac{17101}{810} \pi^{2}+\frac{139}{16} g_{3}^{6}-\frac{149}{9} g_{1}^{6} \pi^{2}\right. \\
& \left.-\frac{525}{32} g_{1}^{6} g_{3}^{6}+\frac{1969}{8640} \pi^{4}-\frac{1161}{128} g_{5}^{6}+\frac{83}{64} g_{3}^{6} \pi^{2}\right)\left(\frac{\alpha}{\pi}\right)^{3} .
\end{aligned}
$$

In the $f$ alphabet, the Galois coaction (4) is deconcatenation. It is therefore easy to read off the Galois conjugates on the right-hand side of the tensor product in the coaction. Up to weight three, we only have the four Galois conjugates

$$
1, \quad \pi^{2}, \quad g_{3}^{6}, \quad g_{1}^{6} \pi^{2} .
$$

Although this list follows from a three loop result it can be conjectured that the list is complete to all loop orders (see e.g. $[15,16])$. In general we expect that one is able to extract the complete list of Galois conjugates of weight $\leq n$ from an $n$ loop result.

In [4] Laporta presents an explicit result for the hyperlogarithmic part of the fourth-order $a_{e}$. The conversion into the $f$ alphabet is given in [63]. It is similar to (29) with additional extensions of MZVs by fourth roots of unity. With $f_{2}^{4} \cong 2 \mathrm{ImLi}_{2}(\mathrm{i})$ we obtain the following six Galois conjugates of weight 4 :

$$
g_{4}^{6}, \quad g_{1}^{6} g_{3}^{6}, \quad g_{2}^{6} \pi^{2}, \quad f_{2}^{4} \pi^{2}, \quad g_{1}^{6} g_{1}^{6} \pi^{2}, \quad \pi^{4} .
$$

The result is preliminary because it does not contain the Galois conjugates of the nonhyperlogarithmic part of $a_{e}$. It is also possible that Laporta's result misses some hyperlogarithmic terms (hidden in the nonhyperlogarithmic part).
Because the coaction conjectures basically work graph by graph, it still makes sense to analyze the partial result [63].

The two most remarkable properties of the motivic structure of $a_{e}$ are

(1) The $\mathbb{Q}$ vector spaces of Galois conjugates at given weight have very low dimensions. This is a strong sparsity property of QED, similar to the one found for $\mathcal{P}_{\phi^{4}}$.

(2) The type of numbers in $a_{e}$ corresponds to what we found in $\mathcal{P}_{\phi^{4}}$. We have MZVs $\left(g_{3}^{6}, g_{5}^{6}\right)$, Euler sums $\left(g_{1}^{6}, g_{1}^{6} g_{1}^{6}, g_{1}^{6} g_{3}^{6}\right)$, extensions by sixth roots of unity $\left(g_{2}^{6}, g_{4}^{6}\right)$, and extensions by fourth roots of unity $\left(f_{2}^{4}\right)$. These correspond to the $c_{2}$ invariants $-1,-z_{2},-z_{3}$, $-z_{4}$ found in $\phi^{4}$ theory. There seems to exist nothing else in the polylogarithmic part of $a_{e}$ up to loop-order four. The only difference to massless $\phi^{4}$ theory is that the numbers come at smaller loop orders, namely 2,2,4,4 for $a_{e}$ in contrast to $3,9,7,8$ for $\phi^{4}$.

\section{ACKNOWLEDGMENTS}

I am very grateful to D. Kreimer and F. Knop for their support. I am also deeply indebted to my coauthors F. Brown, E. Panzer, and K. Yeats. The calculation of the seven-loop renormalization functions used some input from E. Panzer's Maple package HyperInt. He also supported the author with very valuable discussions. Many results in this report were found when the author was visiting scientist at the Humboldt University, Berlin. The author is supported by DFG Grant No. SCHN 1240/2. The computer calculations were performed on the server mem of the Department Mathematik, Friedrich-Alexander Universität of Erlangen-Nürnberg.
[1] T. Aoyama, M. Hayakawa, T. Kinoshita, and M. Nio, Revised value of the eighth-order QED contribution to the anomalous magnetic moment of the electron, Phys. Rev. D 77, 053012 (2008).

[2] D. Hanneke, S. Fogwell, and G. Gabrielse, New Measurement of the Electron Magnetic Moment and the Fine Structure Constant, Phys. Rev. Lett. 100, 120801 (2008).

[3] S. Laporta and E. Remiddi, The analytical value of the electron $(g-2)$ at order $\alpha^{3}$ in QED, Phys. Lett. B 379, 283 (1996).

[4] S. Laporta, High-precision calculation of the 4-loop contribution to the electron $g-2$ in QED, Phys. Lett. B 772, 232 (2017).

[5] F. C. S. Brown and D. Doryn, Framings for graph hypersurfaces, arXiv:1301.3056v1 (2013).
[6] F. C. S. Brown and O. Schnetz, A K3 in $\phi^{4}$, Duke Math. J. 161, 1817 (2012).

[7] J. Drummond, C. Duhr, P. Heslop, J. Pennington, and V. A. Smirnov, Leading singularities and off-shell conformal integrals, J. High Energy Phys. 08 (2013) 133.

[8] M. Golz, E. Panzer, and O. Schnetz, Graphical functions in parametric space, Lett. Math. Phys. 107, 1177 (2017).

[9] O. Schnetz, Graphical functions and single-valued multiple polylogarithms, Comm. Number Theory Phys. 8, 589 (2014).

[10] D. Broadhurst and D. Kreimer, Knots and numbers in $\phi^{4}$ theory to 7 loops and beyond, Int. J. Mod. Phys. C 06, 519 (1995).

[11] F. C. S. Brown and O. Schnetz, Single-valued multiple polylogarithms and a proof of the zig-zag conjecture, J. Number Theory 148, 478 (2015). 
[12] M. Kontsevich and D. Zagier, Periods, in Mathematics Unlimited -2001 and Beyond, edited by B. Engquis and W. Schmid (Springer, New York, 2001).

[13] O. Schnetz, Generalized single-valued hyperlogarithms (to be published).

[14] O. Schnetz, HyperlogProcedures, Maple package available on the homepage of the author.

[15] F. C. S. Brown, Feynman amplitudes, coaction principle, and cosmic Galois group, Comm. Number Theory and Physics 11, 453 (2017).

[16] F. C. S. Brown, Notes on motivic periods, Comm. Number Theory Phys. 11, 557 (2017).

[17] E. Panzer and O. Schnetz, The Galois coaction on $\phi^{4}$ periods, Comm. Number Theory Phys. 11, 657 (2017).

[18] J. C. Itzykson and J. B. Zuber, Quantum Field Theory (Mc-Graw-Hill, New York, 1980).

[19] O. Schnetz, The geometry of one-loop amplitudes, arXiv: 1010.5334 (2010).

[20] N. Nakanishi, Feynman-parametric formula for the Hankeltransformed position-space Feynman integral, Prog. Theor. Phys. 42, 966 (1969).

[21] N. Nakanishi, Graph theory and Feynman integrals, in Mathematics and Its Applications (Gordon and Breach, New York, 1971), Vol. 11.

[22] O. Schnetz, Quantum periods: A census of $\phi^{4}$ transcendentals, Comm. Number Theory Phys. 4, 1 (2010).

[23] E. Panzer, Ph.D. thesis, Humboldt-Universität zu Berlin, arXiv:1506.07243 (2014).

[24] K. G. Chetyrkin, A. L. Kataev, and F. V. Tkachov, New approach to evaluation of multiloop Feynman integrals: The Gegenbauer polynomial $x$-space technique, Nucl. Phys. B174, 345 (1980).

[25] K. G. Chetyrkin and F. V. Tkachov, Integration by parts: The algorithm to calculate $\beta$-functions in 4 loops, Nucl. Phys. B192, 159 (1981).

[26] D. I. Kazakov, The method of uniqueness, a new powerful technique for multiloop calculations, Phys. Lett. B 133, 406 (1983).

[27] N. I. Ussyukina, Calculation of multiloop diagrams in high orders of perturbation theory, Phys. Lett. B 267, 382 (1991).

[28] O. Schnetz, Calculation of the $\phi^{4}$ 6-loop non-zeta transcendental, arXiv:hep-th/9912149 (1999).

[29] D. Broadhurst, Multiple zeta values and other periods in quantum field theory, in Proc. Bristol, 2011.

[30] E. Panzer (unpublished).

[31] D. Broadhurst, Multiple Deligne values: A data mine with empirically tamed denominators, arXiv:1409.7204 (2014).

[32] F. C. S. Brown and O. Schnetz, Modular forms in quantum field theory, Comm. Number Theory Phys. 7, 293 (2013).

[33] Y. André, Galois theory, motives and transcendental numbers, in Renormalization and Galois theories, 15 of IRMA Lect. Math. Theor. Phys., Eur. Math. Soc., Zürich, 165-177 (2009).

[34] F. C. S. Brown, Mixed Tate motives over $\mathbb{Z}$, Ann. Math. 175, 949 (2012).

[35] F. C. S. Brown, On the decomposition of motivic multiple zeta values, in Galois-Teichmüller Theory and Arithmetic Geometry, Advanced Studies in Pure Mathematics (Tokyo) (Mathematical Society of Japan, 2012), Vol. 68, p. 31.
[36] F. C. S. Brown, Single-valued periods and multiple zeta values, Forum of Mathematics, Sigma 2, e25 (2014).

[37] F. C.S. Brown, Motivic periods and the projective line minus three points, Proceedings of the ICM 2014 MSCclass: 11M32, 14C15, arXiv:1407.5165 (2014).

[38] A. B. Goncharov, Galois symmetries of fundamental groupoids and noncommutative geometry, Duke Math. J. 128, 209 (2005).

[39] O. Schnetz, Quantum field theory over $\mathbb{F}_{q}$, Electron. J. Comb. 18N1, P102 (2011).

[40] F. C. S. Brown, O. Schnetz, and K. Yeats, Properties of $c_{2}$ invariants of Feynman graphs, Adv. Theor. Math. Phys. 18, 323 (2014).

[41] D. Doryn, The $c_{2}$ invariant is invariant, arXiv:1312.7271 (2013).

[42] K. Yeats, A few $c_{2}$ invariants of circulant graphs, Comm. Number Theory Phys. 10, 63 (2016).

[43] M. Kompaniets and E. Panzer, Minimally subtracted six loop renormalization of $O(n)$-symmetric $\phi^{4}$ theory and critical exponents, Phys. Rev. D 96, 036016 (2017).

[44] D. Zagier, The dilogarithm function in geometry and number theory, in Number theory and related topics, Proceedings of the Ramanujan Colloquium, Bombay 1988, Studies in Mathematics 12, (TIFR and Oxford University Press, New York, 1989), p. 231; The dilogarithm function in geometry and number theory, J. Math. Phys. Sci. 22, 131 (1988).

[45] O. Schnetz, Graphical functions (to be published).

[46] M. Golz, Master's thesis, www2.mathematik.hu-berlin .de/ kreimer/wp-content/uploads/GolzMasterThesis.pdf (2015).

[47] F. C. S. Brown, Single-valued multiple polylogarithms in one variable, C. R. Acad. Sci. Paris, Ser. I 338, 527 (2004).

[48] F. C. S. Brown, The massless higher-loop two-point function, Commun. Math. Phys. 287, 925 (2009).

[49] F. C. S. Brown, On the periods of some Feynman integrals, arXiv:0910.0114 (2009).

[50] E. Panzer, Algorithms for the symbolic integration of hyperlogarithms with applications to Feynman integrals, Comput. Phys. Commun. 188, 148 (2015).

[51] B. Basso and L. J. Dixon, Gluing Ladder Feynman Diagrams into Fishnets, Phys. Rev. Lett. 119, 071601 (2017).

[52] K. Chen, Algebras of iterated path integrals and fundamental groups, Trans. Am. Math. Soc. 156, 359 (1971).

[53] F. Chavez and C. Duhr, Three-mass triangle integrals and single-valued polylogarithms, J. High Energy Phys. 11 (2012) 114.

[54] S. Weinberg, High-energy behavior in quantum field theory, Phys. Rev. 118, 838 (1960).

[55] S. Bloch, H. Esnault, and D. Kreimer, On motives associated with graph polynomials, Commun. Math. Phys. 267, 181 (2006).

[56] P. A. Baikov and K. G. Chetyrkin, Four loop massless propagators: An algebraic evaluation of all master integrals, Nucl. Phys. B837, 186 (2010).

[57] R. N. Lee, A. V. Smirnov, and V. A. Smirnov, Master integrals for four-loop massless propagators up to weight twelve, Nucl. Phys. B856, 95 (2012). 
[58] O. Schnetz, Seven loops $\phi^{4}$ (to be published).

[59] M. Borinsky, Feynman graph generation and calculations in the Hopf algebra of Feynman graphs, Comput. Phys. Commun. 185, 3317-3330 (2014).

[60] H. Kleinert, J. Neu, V. Schulte-Frohlinde, K. G. Chetyrkin, and S. A. Larin, Five-loop renormalization group functions of $O(n)$-symmetric $\phi^{4}$-theory and $\epsilon$-expansions of critical exponents up to $\epsilon^{5}$, Phys. Lett. B 272, 39 (1991); Erratum, Phys. Lett. B 319, 545(E) (1993).
[61] D. V. Batkovich, K. G. Chetyrkin, and M. V. Kompaniets, Six loop analytical calculation of the field anomalous dimension and the critical exponent $\eta$ in $O(n)$-symmetric $\phi^{4}$ model, Nucl. Phys. B906, 147 (2016).

[62] D. Broadhurst, Multi-loop calculations without subtractions: 5-loop propagator in $\phi^{4}$ theory, unpublished notes OUT-4102-46 (1993).

[63] O. Schnetz, The Galois coaction on the electron anomalous magnetic moment, arXiv:1711.05118 (2017). 